\title{
Global Existence for the Relativistic Vlasov-Maxwell System with Nearly Neutral Initial Data
}

\author{
R. T. Glassey ${ }^{1}$ and J. W. Schaeffer ${ }^{2}$ \\ 1 Indiana University, Bloomington, Indiana 47405, USA \\ 2 Carnegie Mellon University, Pittsburgh, Pennsylvania 15213, USA
}

\begin{abstract}
Global classical solutions to the initial value problem for the relativistic Vlasov-Maxwell equations are obtained in three space dimensions. The initial distribution of the various species may be large, provided that the total positive charge nearly cancels the total negative charge.
\end{abstract}

\section{Introduction}

Consider a plasma which consists of a large number of charged particles. We assume there are $N$ distinct species of charge and that particles of the $\alpha^{\text {th }}$ species have charge $e_{\alpha}$ and rest mass $m_{\alpha}$. Their distribution in phase space at time $t$ is $f_{\alpha}(t, x, v)$, where $x \in \mathbb{R}^{3}$ represents position and $v \in \mathbb{R}^{3}$ momentum. Thus the charge and current densities are

and

$$
\rho(t, x)=4 \pi \int\left(\sum_{\alpha} e_{\alpha} f_{\alpha}\right) d v
$$

where

$$
j(t, x)=4 \pi \int\left(\sum_{\alpha} e_{\alpha} f_{\alpha} \hat{v}_{\alpha}\right) d v
$$

$$
\hat{v}_{\alpha}=\left(m_{\alpha}^{2}+c^{-2}|v|^{2}\right)^{-1 / 2} v
$$

gives the velocity of a particle with momentum $v$ and rest mass $m_{\alpha}(c$ is the speed of light). The state of the plasma at time $t$ is given by $f_{\alpha}(t, \cdot, \cdot)$ for $\alpha=1, \ldots, N$ and $E(t, \cdot), B(t, \cdot)$, where $E$ and $B$ are the electric and magnetic fields. If electromagnetic effects dominate collisional effects, we may model the time evolution of the plasma with the system:

$$
(\mathrm{RVM})\left\{\begin{array}{l}
\partial_{t} f_{\alpha}+\hat{v}_{\alpha} \cdot \nabla_{x} f_{\alpha}+e_{\alpha}\left(E+c^{-1} \hat{v}_{\alpha \Lambda} B\right) \cdot \nabla_{v} f_{\alpha}=0 \\
\partial_{t} E=c \nabla_{\Lambda} B-j, \quad \nabla \cdot E=\rho \\
\partial_{t} B=-c \nabla_{\Lambda} E, \quad \nabla \cdot B=0
\end{array}\right.
$$

\footnotetext{
1 Research supported in part by NSF DMS 85-20662

${ }^{2}$ Research supported in part by NSF DMS 86-02952
} 
with the initial data

$$
\left\{\begin{array}{c}
f_{\alpha}(0, x, v)=f_{\alpha}^{0}(x, v) \\
E(0, x)=E^{0}(x) \\
B(0, x)=B^{0}(x) .
\end{array}\right.
$$

The goal here is to find conditions on the initial data which ensure the existence of a smooth solution of (RVM) for all time, thus ruling out shock waves and other singularities. Two results in this direction are due to Glassey and Strauss: In [4] it is shown that solutions may be continued as long as the $v$-support of each $f_{\alpha}$ remains finite on every finite time interval. In [5] global existence and decay in time are shown for a class of "small" data. The result of [5] requires that

$$
\left\|f_{\alpha}^{0}\right\|_{c^{0}\left(\mathbb{R}^{6}\right)}+\left\|\nabla f_{\alpha}^{0}\right\|_{c^{0}\left(\mathbb{R}^{6}\right)}
$$

(we'll denote this by $\left\|f_{\alpha}^{0}\right\|_{c^{1}}$ ) be small for each $\alpha$, hence the total positive (and negative) charge must be small. Here we generalize this result by requiring a smallness condition which allows the total positive charge to be large provided the negative charge nearly cancels it. Thus the result of this paper applies to more realistic physical cases than that of [5]. Unfortunately the method of [5] does not suffice for this result and a substantial new element is required.

More is known about the Poisson-Vlasov system (than (RVM)) in which magnetic effects are ignored. The interested reader is referred to the survey [2] and also to $[1,3$ and 6$]$.

\section{The Main Result}

In order to correctly state the appropriate neutrality condition we reindex the problem in the following way: Define

$$
g_{\alpha}(t, x, u)=f_{\alpha}\left(t, x, m_{\alpha} u\right)
$$

and

$$
g_{\alpha}^{0}(x, u)=f_{\alpha}^{0}\left(x, m_{\alpha} u\right)
$$

We define

$$
\hat{u}=\left(1+c^{-2}|u|^{2}\right)^{-1 / 2} u
$$

for any $u \in \mathbb{R}^{3}$. By making the change of variables $v=m_{\alpha} u$ we see that

$$
\int f_{\alpha}^{0}(x, v) d v=m_{\alpha}^{3} \int g_{\alpha}^{0}(x, u) d u .
$$

Similarly

and

$$
\rho(t, x)=4 \pi \int\left(\sum_{\alpha} e_{\alpha} m_{\alpha}^{3} g_{\alpha}(t, x, u)\right) d u
$$

$$
j(t, x)=4 \pi \int\left(\sum_{\alpha} e_{\alpha} m_{\alpha}^{3} g_{\alpha}(t, x, u)\right) \hat{u} d u .
$$

Thus we will require $\sum_{\alpha} e_{\alpha} m_{\alpha}^{3} g_{\alpha}$ to be small. 
For $k, M$, and $\varepsilon$ positive define

$$
\begin{aligned}
& \mathscr{D}(k, M, \varepsilon)=\left\{\left(g_{1}^{0}, \ldots, g_{N}^{0}, E^{0}, B^{0}\right):\right. \\
& g_{\alpha}^{0} \in C_{0}^{2}\left(\mathbb{R}^{6}\right) ; \quad E^{0}, B^{0} \in C_{0}^{2}\left(\mathbb{R}^{3}\right) ; \quad g_{\alpha}^{0} \geqq 0 ; \quad g_{\alpha}^{0}(x, v)=0 \\
& \text { if } \quad|x| \geqq k \quad \text { or } \quad|v| \geqq k ; \quad E^{0}(x)=B^{0}(x)=0 \quad \text { if } \quad|x| \geqq k ; \\
& \nabla \cdot B^{0}=0 \quad \text { and } \quad \nabla \cdot E^{0}=4 \pi \int\left(\sum_{\alpha} e_{\alpha} m_{\alpha}^{3} g_{\alpha}^{0}\right) d u ; \\
& \sum_{\alpha}\left\|g_{\alpha}^{0}\right\|_{c^{2}}<M ; \\
& \left.\left\|\sum_{\alpha} e_{\alpha} m_{\alpha}^{3} g_{\alpha}^{0}\right\|_{c^{1}}+\left\|E^{0}\right\|_{c^{2}}+\left\|B^{0}\right\|_{c^{2}}<\varepsilon\right\} .
\end{aligned}
$$

Theorem. For any $k$ and $M$ positive there exists $\varepsilon=\varepsilon(k, M)>0$ such that if $\left(g_{1}^{0}, \ldots, g_{N}^{0}, E^{0}, B^{0}\right) \in \mathscr{D}(k, M, \varepsilon(k, M))$, then (RVM) with initial data $\left(f_{1}^{0}, \ldots, f_{N}^{0}, E^{0}, B^{0}\right)$ has a unique continuously differentiable solution for all $t \geqq 0$. Here $f_{\alpha}^{0}$ is given by

$$
f_{\alpha}^{0}(x, v)=g_{\alpha}^{0}\left(x, m_{\alpha}^{-1} v\right) .
$$

Moreover, for $|x|<t+k$,

and

$$
|E(t, x)|+|B(t, x)| \leqq C(1+k+t+|x|)^{-3 / 4}(1+k+t-|x|)^{-3 / 4}
$$

$$
\left|\nabla_{x} E(t, x)\right|+\left|\nabla_{x} B(t, x)\right| \leqq C(1+k+t+|x|)^{-3 / 4}(1+k+t-|x|)^{-7 / 4} .
$$

\section{An Outline of the Proof}

Consider data in $\mathscr{D}(k, M, \varepsilon)$. Define

$$
\mathscr{X}(t, x)=\left\{\begin{array}{lll}
1 & \text { if } & |x|<k+t \\
0 & \text { if } & |x| \geqq k+t .
\end{array}\right.
$$

It follows from the continuation result of [4] that a unique continuously differentiable solution exists on some time interval. For $\eta>\varepsilon$ the estimate

$$
(\mathrm{FSC})\left\{\begin{array}{l}
|E|+|B| \leqq \eta(1+k+t+|x|)^{-3 / 4}(1+k+t-|x|)^{-3 / 4} \mathscr{X}(t, x) \\
\left|\nabla_{x} E\right|+\left|\nabla_{x} B\right| \leqq \eta(1+k+t+|x|)^{-3 / 4}(1+k+t-|x|)^{-7 / 4} \mathscr{X}(t, x)
\end{array}\right.
$$

will hold for some time interval. The idea is that the (FSC) ensures that the motion which occurs is essentially "free streaming"; that is, uninfluenced by $E$ and $B$. The proof consists mainly of a sequence of estimates which show that if $\varepsilon$ is sufficiently small, then the (FSC) (with an appropriate choice of $\eta$ ) holds on $[0, \infty)$. The first set of estimates use the neutrality assumption, i.e.

$$
\left\|\sum_{\alpha} e_{\alpha} m_{\alpha}^{3} g_{\alpha}^{0}\right\|_{c^{1}}+\left\|E^{0}\right\|_{c^{2}}+\left\|B^{0}\right\|_{c^{2}}<\varepsilon
$$

to show that the (FSC) persists for a long time if $\varepsilon$ is small. This is essentially stability analysis on bounded time intervals. The second set of estimates are similar to those in the small data result [5]. The structure of the argument is then roughly as follows: For $\varepsilon$ small the (FSC) must hold for a long time, during which the 
solution disperses. By taking $\varepsilon$ smaller we force the dispersion to be more complete. Once the dispersion is sufficiently complete, the (FSC) holds by virtue of the dispersion only. This line of reasoning is similar to that used in [7].

\section{Preliminaries}

Define the characteristics $\mathscr{X}_{\alpha}(s, t, x, v)$ and $\mathscr{V}_{\alpha}(s, t, x, v)$, induced by $E$ and $B$, by

$$
\left\{\begin{array}{l}
\dot{\mathscr{X}}_{\alpha}=\widehat{\mathscr{V}}_{\alpha}, \quad \mathscr{X}_{\alpha}(t, t, x, v)=x \\
\dot{\mathscr{V}}_{\alpha}=e_{\alpha}\left(E\left(s, \mathscr{X}_{a}\right)+c^{-1} \hat{\mathscr{V}}_{\alpha} \wedge B\left(s, \mathscr{X}_{\alpha}\right)\right), \quad \mathscr{V}_{\alpha}(t, t, x, v)=v .
\end{array}\right.
$$

It follows from (RVM) that

$$
f_{\alpha}(t, x, v)=f_{\alpha}^{0}\left(\mathscr{X}_{\alpha}(0, t, x, v), \mathscr{V}_{\alpha}(0, t, x, v)\right),
$$

hence also

$$
\begin{aligned}
g_{\alpha}\left(t, x, m_{\alpha}^{-1} v\right) & =f_{\alpha}(t, x, v)=f_{\alpha}^{0}\left(\mathscr{X}_{\alpha}(0, t, x, v), \mathscr{V}_{\alpha}(0, t, x, v)\right) \\
& =g_{\alpha}^{0}\left(\mathscr{X}_{\alpha}(0, t, x, v), m_{\alpha}^{-1} \mathscr{V}_{\alpha}(0, t, x, v)\right)
\end{aligned}
$$

or equivalently

$$
g_{\alpha}(t, x, u)=g_{\alpha}^{0}\left(\mathscr{X}_{\alpha}\left(0, t, x, m_{\alpha} u\right), m_{\alpha}^{-1} \mathscr{V}_{\alpha}\left(0, t, x, m_{\alpha} u\right)\right)
$$

Thus $g_{\alpha}$ and $f_{\alpha}$ remain nonnegative.

Constants which may change from line to line are denoted by $C$. These may depend on $k, M, e_{\alpha}$, and $m_{\alpha}$, but not on $\varepsilon$. Similarly $C(t)$ denotes the value of an increasing locally bounded function from $[0, \infty)$ to $[0, \infty)$. Also $\|E(t)\|_{c^{k}}$ and $\left\|f_{\alpha}(t)\right\|_{c^{k}}$ denote the $C^{k}$ norms of the mappings $x \mapsto E(t, x)$ and $(x, v) \mapsto f_{\alpha}(t, x, v)$ respectively. Henceforth the speed of light, $c$, is taken to be 1 . For brevity we will write

$$
K(t, x, u)=E(t, x)+\hat{u} \wedge B(t, x) .
$$

where " $\wedge$ " denotes the ordinary vector cross product. We will also write

$$
|K(t, x)|=|E(t, x)|+|B(t, x)|
$$

when no confusion may arise. The Lebesgue surface measure on the unit sphere in $\mathbb{R}^{3}$ is denoted " $d w$."

\section{Consequences of (FSC)}

The following two lemmas will allow us to estimate the support of $f_{\alpha}(t, \cdot, \cdot)$ as long as the (FSC) holds.

Lemma 1. There exists $\eta_{1}>0$ such that if the (FSC) holds for $0 \leqq t<T$ with $\eta \leqq \eta_{1}$, then the following holds: If $|x| \leqq k,|v| \leqslant k$, and $0 \leqq t<T$ then (for each $\alpha$ )

and

$$
\left|\mathscr{V}_{\alpha}(t, 0, x, v)\right| \leqq 2 k,
$$

with $c_{1}<1$.

$$
\left|\mathscr{X}_{\alpha}(t, 0, x, v)\right| \leqq k+c_{1} t
$$


Proof. Assume the (FSC) holds on [0,T). Fix $\alpha, x$, and $v$ with $|x|<k$ and $|v|<k$. Define

$$
T_{1}=\sup \left\{t \in[0, T):\left|\mathscr{V}_{\alpha}(s, 0, x, v)\right| \leqq 2 k \text { for all } s \in[0, t]\right\} .
$$

Now for $t \in\left[0, T_{1}\right)$

$$
\left|\mathscr{X}_{\alpha}(t, 0, x, v)\right| \leqq k+\int_{0}^{t}\left|\widehat{\mathscr{V}}_{\alpha}(s, 0, x, v)\right| d s \leqq k+\frac{2 k}{\sqrt{m_{\alpha}^{2}+(2 k)^{2}}} t
$$

so by the (FSC)

$$
\begin{aligned}
\left|E\left(t, \mathscr{X}_{\alpha}\right)+\widehat{\mathscr{V}}_{\alpha} \wedge B\left(t, \mathscr{X}_{\alpha}\right)\right| & \leqq C \eta\left(1+k+t+\left|\mathscr{X}_{\alpha}\right|\right)^{-3 / 4}\left(1+k+t-\left|\mathscr{X}_{\alpha}\right|\right)^{-3 / 4} \\
& \leqq C \eta(1+2 k+C t)^{-3 / 4}\left(1+\left[1-\frac{2 k}{\sqrt{m_{\alpha}^{2}+4 k^{2}}}\right] t\right)^{-3 / 4} \\
& \leqq C \eta(1+t)^{-3 / 2}
\end{aligned}
$$

Thus for $t \in\left[0, T_{1}\right)$

$$
\left|\mathscr{V}_{\alpha}(t, 0, x, v)\right| \leqq k+\int_{0}^{t} C \eta(1+\tau)^{-3 / 2} d \tau \leqq k+2 C \eta
$$

Now if $\eta$ is taken sufficiently small, (2) says that

$$
\left|\mathscr{V}_{\alpha}(t, 0, x, v)\right| \leqq \frac{3}{2} k
$$

and it follows that $T_{1}=T$. Now the lemma follows by (1) and (2).

Definition.

$$
\begin{gathered}
\mathscr{S}=\underset{\alpha}{\mathrm{U}}\left\{\left(t, \mathscr{X}_{\alpha}(t, 0, x, v), \mathscr{V}_{a}(t, 0, x, v)\right):\right. \\
t \geqq 0, \quad|x| \leqq k, \quad \text { and } \quad|v| \leqq k\},
\end{gathered}
$$

so that support $f_{\alpha} \subset \mathscr{S}$. Also define

$$
\mathscr{P}(t, x)=\mathrm{U}_{\alpha}\left\{\mathscr{V}_{\alpha}(t, 0, y, v):|y| \leqq k,|v| \leqq k, \quad \text { and } \quad \mathscr{X}_{\alpha}(t, 0, y, v)=x\right\}
$$

for each $t \geqq 0$ and $x \in \mathbb{R}^{3}$, so that

$$
\int f_{\alpha}(t, x, v) d v=\int_{\mathscr{S}(t, x)} f_{\alpha}(t, x, v) d v
$$

Lemma 2. There exists $\eta_{2} \in\left(0, \eta_{1}\right)$ such that if the (FSC) holds for $0 \leqq t<T$ with $\eta \leqq \eta_{2}$, then the following holds:

$$
\left|\mathscr{X}_{\alpha}(0, t, x, v)-\mathscr{X}_{\alpha}(0, t, x, w)\right| \geqq C t|v-w|
$$

for $t \in[0, T),(t, x, v)$ and $(t, x, w) \in \mathscr{S}$, and $\alpha \in\{1, \ldots, N\}$.

Proof. Assume the (FSC) holds on $[0, T)$ with $\eta \in\left(0, \eta_{1}\right)$. We may write (for $t \in[0, T)$ )

$$
\mathscr{X}_{\alpha}(s, t, x, v)=\mathscr{X}_{\alpha}\left(s, 0, \mathscr{X}_{\alpha}(0, t, x, v), \mathscr{V}_{\alpha}(0, t, x, v)\right)
$$

and similarly for $\mathscr{V}_{\alpha}$. Since $(t, x, v) \in \mathscr{S},\left|\mathscr{X}_{a}(0, t, x, v)\right| \leqq k$ and $\left|\mathscr{V}_{\alpha}(0, t, x, v)\right| \leqq k$, so 
Lemma 1 applies. Now by Lemma 1 and the (FSC),

$$
\begin{aligned}
& \left|E\left(s, \mathscr{X}_{\alpha}(s, t, x, v)\right)\right|+\left|B\left(s, \mathscr{X}_{\alpha}(s, t, x, v)\right)\right| \\
& \quad \leqq \eta(1+k+s)^{-3 / 4}\left(1+k+s-\left[k+c_{1} s\right]\right)^{-3 / 4} \\
& \quad \leqq C \eta(1+s)^{-3 / 2} \equiv h(s)
\end{aligned}
$$

and

$$
\left|\nabla_{x} E\left(s, \mathscr{X}_{\alpha}(s, t, x, v)\right)\right|+\left|\nabla_{x} B\left(s, \mathscr{X}_{\alpha}(s, t, x, v)\right)\right| \leqq C \eta(1+s)^{-5 / 2} \equiv g(s) .
$$

By the same reasoning (3) and (4) hold if we replace $v$ by $w$. Now the proof is the same as that of Lemma 3 of [5], except that the decay rates in (3) and (4) are slower than those in [5]. Even with the slower decay rates, the proof in [5] works, the key point being that

$$
\lim \int_{n \backslash 0}^{\infty}[s g(s)+h(s)] d s=0 .
$$

Lemma 3. Assume the (FSC) holds on $[0, T)$ with $\eta \in\left(0, \eta_{2}\right]$. Then for all $(t, x) \in[0, T) \times \mathbb{R}^{3}$ the Lebesgue measure of $\mathscr{S}(t, x)$ is less than $C(1+t)^{-3}$.

Proof. Let us denote

$$
S_{\alpha}=\left\{v:\left|\mathscr{V}_{\alpha}(0, t, x, v)\right| \leqq k \quad \text { and } \quad\left|\mathscr{X}_{\alpha}(0, t, x, v)\right| \leqq k\right\}
$$

for a given $(t, x)$. Note that

$$
\mathscr{S}(t, x)=\mathrm{U}_{\alpha} S_{\alpha}
$$

If $\left|\mathscr{V}_{\alpha}(0, t, x, v)\right| \leqslant k$ and $\left|\mathscr{X}_{\alpha}(0, t, x, v)\right| \leqq k$, then by Lemma 1 ,

$$
|v|=\left|\mathscr{V}_{\alpha}\left(t, 0, \mathscr{X}_{\alpha}(0, t, x, v), \mathscr{V}_{\alpha}(0, t, x, v)\right)\right| \leqq 2 k .
$$

Hence

$$
S_{\alpha} \subset\{v:|v| \leqq 2 k\} .
$$

Now suppose also that $\left|\mathscr{V}_{\alpha}(0, t, x, w)\right| \leqq k$ and $\left|\mathscr{X}_{\alpha}(0, t, x, w)\right| \leqq k$. Then by Lemma 2 ,

$$
2 k \geqq\left|\mathscr{X}_{\alpha}(0, t, x, v)-\mathscr{X}_{\alpha}(0, t, x, w)\right| \geqq C t|v-w| .
$$

Hence $S_{\alpha}$ is contained in a ball of radius $\min \left\{2 k, 2 k(C t)^{-1}\right\}$ and the lemma follows. Comment. Lemma 3 will be used repeatedly. To illustrate how it will be used we observe that

$$
|\rho(t, x)| \leqq \int_{\mathscr{S}(t, x)} \sum_{\alpha}\left|e_{\alpha}\right| f_{\alpha}(t, x, v) d v \leqq C(1+t)^{-3},
$$

as long as the (FSC) holds.

\section{Representation of the Fields}

We begin with the representation formula for $E$ and $B$ from Theorem 3 of [4]. Let

$$
w=|y-x|^{-1}(y-x)
$$


and let $\mathscr{E}$ be the solution of

$$
\left\{\begin{array}{l}
\mathscr{E}_{t t}-\Delta \mathscr{E}=0 \\
\mathscr{E}(0, x)=E^{0}(x) \\
\mathscr{E}_{t}(0, x)=\nabla \wedge B^{0}(x)-j(0, x) .
\end{array}\right.
$$

Note that by (RVM)

$$
\left.\left[\partial_{t} g_{\alpha}+\hat{u} \cdot \nabla_{x} g_{\alpha}+\nabla_{u} \cdot\left(e_{\alpha} m_{\alpha}^{-1} K g_{\alpha}\right)\right]\right|_{(t, x, u)}=0 .
$$

Now following [4] we take

and

$$
T=\nabla_{x}-w \partial_{t}
$$

and obtain

$$
S=\partial_{t}+\hat{u} \cdot \nabla_{x}
$$

where

$$
E=E_{z}+E_{T}+E_{S}
$$

$$
\begin{aligned}
E_{Z}= & \mathscr{E}-t^{-1} \int_{|x-y|=t} \int(1+\hat{u} \cdot w)^{-1}(w-(\hat{u} \cdot w) \hat{u})\left(\sum_{\alpha} e_{\alpha} m_{\alpha}^{3} g_{\alpha}^{0}(y, u)\right) d u d S_{y} \\
E_{T}= & -\int_{|x-y|<t}|x-y|^{-2} \int(1+\hat{u} \cdot w)^{-2}\left(1-|\hat{u}|^{2}\right)(w+\hat{u}) \\
& \cdot\left(\sum_{\alpha} e_{\alpha} m_{\alpha}^{3} g_{\alpha}(t-|x-y|, y, u)\right) d u d y
\end{aligned}
$$

and

$$
E_{S}=-\left.\int_{|x-y|<t}|x-y|^{-1} \int(1+\hat{u} \cdot w)^{-1}(w+\hat{u})\left(\sum_{\alpha} e_{\alpha} m_{\alpha}^{3} S g_{\alpha}\right)\right|_{(t-|x-y|, y, u)} d u d y .
$$

By (5)

$$
S g_{\alpha}=-\nabla_{u} \cdot\left(e_{\alpha} m_{\alpha}^{-1} K g_{\alpha}\right),
$$

so, integrating by parts we obtain

$$
E_{S}=-\left.\int_{|x-y|<t}|x-y|^{-1} \int \nabla_{u}\left([1+\hat{u} \cdot w]^{-1}[w+\hat{u}]\right)\left(\sum_{\alpha} e_{\alpha}^{2} m_{\alpha}^{2} g_{\alpha}\right) K\right|_{(t-|x-y|, y, u)} d u d y
$$

In the same fashion

where

$$
B=B_{z}+B_{T}+B_{S},
$$

$$
\begin{aligned}
B_{z}= & \mathscr{B}+t^{-1} \int_{|x-y|=t} \int(1+\hat{u} \cdot w)^{-1}(w \wedge \hat{u})\left(\sum_{\alpha} e_{\alpha} m_{\alpha}^{3} g_{\alpha}^{0}(y, u)\right) d u d S_{y} . \\
B_{T}= & \int_{|x-y|<t}|x-y|^{-2} \int(1+\hat{u} \cdot w)^{-2}\left(1-|\hat{u}|^{2}\right)(w \wedge \hat{u}) \\
& \cdot\left(\sum_{\alpha} e_{\alpha} m_{\alpha}^{3} g_{\alpha}(t-|x-y|, y, u)\right) d u d y,
\end{aligned}
$$


and

$$
B_{S}=\left.\int_{|x-y|<t}|x-y|^{-1} \int \nabla_{u}\left([1+\hat{u} \cdot w]^{-1}[w \wedge \hat{u}]\right)\left(\sum_{\alpha} e_{\alpha}^{2} m_{\alpha}^{2} g_{\alpha}\right) K\right|_{(t-|x-y|, y, u)} d u d y .
$$

Here $\mathscr{B}$ is defined by the solution of

$$
\left\{\begin{array}{l}
\mathscr{B}_{t t}-\Delta \mathscr{B}=0 \\
\mathscr{B}(0, x)=B^{0}(x) \\
\mathscr{B}_{t}(0, x)=-\nabla \wedge E^{0}(x) .
\end{array}\right.
$$

We point out that the expected cancellation in the sums over $\alpha$ is indeed present in the formulae (8) and (8B) for $E_{T}, B_{T}$, but that this fortunate circumstance does not hold for $E_{S}, B_{S}$ (compare (9), (9B)). Thus the methods of [5] cannot be applied to the terms $E_{S}, B_{S}$.

We will need to estimate derivatives of $E$ and $B$, so we again appeal to [4] to represent the derivatives of $E$ and $B$. By Theorem 4 of [4] we have

where

$$
\frac{\partial E^{i}}{\partial x_{k}}=A_{z}+A_{w}+A_{T T}+A_{T S}+A_{S T}+A_{S S}
$$

$$
\begin{aligned}
A_{T T} & =\int_{|x-y|<t}|y-x|^{-3} \int a(w, \hat{u})\left(\sum_{\alpha} e_{\alpha} m_{\alpha}^{3} g_{\alpha}(t-|x-y|, y, u)\right) d u d y, \\
A_{T S}+A_{S T} & =\left.\int_{|x-y|<t}|y-x|^{-2} \int b(w, \hat{u})\left(\sum_{\alpha} e_{\alpha} m_{\alpha}^{3} S g_{\alpha}\right)\right|_{(t-|x-y|, y, u)} d u d y,
\end{aligned}
$$

and

$$
A_{S S}=\left.\int_{|x-y|<t}|y-x|^{-1} \int c(w, \hat{u})\left(\sum_{\alpha} e_{\alpha} m_{\alpha}^{3} S^{2} g_{\alpha}\right)\right|_{(t-|x-y|, y, u)} d u d y
$$

The kernels $a, b$, and $c$ are given in [4]; they are smooth functions for $|u|$ bounded. Moreover, $a$ has the property that

$$
\int_{|w|=1} a(w, \hat{u}) d w=0
$$

for each $\hat{u} . A_{z}$ is the sum of all the data terms, namely

$$
\begin{aligned}
A_{z}= & \frac{\partial E_{z}}{\partial x_{k}}+t^{-2} \int_{|x-y|=t} \int d(w, \hat{u})\left(\sum_{\alpha} e_{\alpha} m_{\alpha}^{3} g_{\alpha}^{0}(y, u)\right) d u d S_{y} \\
& +t^{-1} \int_{|x-y|=t} \int_{t} e(w, \hat{u})\left(\sum_{\alpha} e_{\alpha} m_{\alpha}^{3} S g_{\alpha}^{0}(y, u)\right) d u d S_{y} .
\end{aligned}
$$

Here $d$ and $e$ are again smooth for $|u|$ bounded. $A_{T T}$ is derived by an integration by parts. Due to the cubic singularity in (11), all integrals are taken over $\varepsilon\langle|x-y|<t$, and then $\varepsilon$ is allowed to tend to zero. This limiting procedure gives rise to the 
term $A_{w}$ :

$$
A_{w}=-\int d(w, \hat{u})\left(\sum_{\alpha} e_{\alpha} m_{\alpha}^{3} g_{\alpha}(t, x, u)\right) d u .
$$

A highly similar representation holds for the derivatives of $B$; we will write it as

$$
\frac{\partial B^{i}}{\partial x_{k}}=\tilde{A}_{z}+\tilde{A}_{w}+\tilde{A}_{T T}+\tilde{A}_{T S}+\tilde{A}_{S T}+\tilde{A}_{S S}
$$

All the data terms may be dealt with at this point.

Lemma 4. For all $(t, x) \in[0, \infty) \times \mathbb{R}^{3}$, we have

$$
\left|E_{z}\right|+\left|B_{z}\right|+\left|A_{z}\right|+\left|\tilde{A}_{z}\right| \leqq C \varepsilon(1+k+t+|x|)^{-1}(1+k+t-|x|)^{-2} \mathscr{X}(t, x),
$$

provided the data is in $\mathscr{D}(k, M, \varepsilon)$.

Proof. It may be shown that

$$
\int_{|x-y|=t}|\psi(y)| d S_{y} \leqq C\|\psi\|_{c^{0}} \min \left\{t^{2}, 1\right\}
$$

for any $\psi \in C_{0}\left(\mathbb{R}^{3}\right)$ for which $\psi(y)=0$ if $|y| \geqq C$. Hence, also for $\phi \in C_{0}^{1}\left(\mathbb{R}^{3}\right)$ with $\phi(y)=0$ if $|y| \geqq C$,

$$
\begin{aligned}
& \left|\frac{\partial}{\partial t}\left(t^{-1} \int_{|x-y|=t} \phi(y) d S_{y}\right)\right| \leqq t^{-2}\left|\int_{|x-y|=t} \phi(y) d S_{y}\right|+t^{-1}\left|\int_{|x-y|=t} \nabla \phi(y) \cdot w d S_{y}\right| \\
& \quad \leqq C\|\phi\|_{c^{1}} \min \left\{1, t^{-1}\right\} .
\end{aligned}
$$

Now since we may solve the homogeneous wave equation for $\mathscr{E}$ as

$$
\mathscr{E}=\frac{1}{4 \pi t} \int_{|x-y|=t} \mathscr{E}_{t}(0, y) d S_{y}+\frac{\partial}{\partial t}\left(\frac{1}{4 \pi t} \int_{|x-y|=t} \mathscr{E}(0, y) d S_{y}\right)
$$

(and similarly for derivatives of $\mathscr{E}$ ) we have by (18) and (19)

$$
\begin{aligned}
|\mathscr{E}| & +\left|\nabla_{x} \mathscr{E}\right| \leqq t^{-1} C\left\|\mathscr{E}_{t}(0)\right\|_{c^{1}} \min \left\{t^{2}, 1\right\}+C\|\mathscr{E}(0)\|_{c^{2}} \min \left\{1, t^{-1}\right\} \\
& \leqq C\left(\left\|\nabla_{\Lambda} B^{0}-j(0)\right\|_{c^{1}}+\left\|E^{0}\right\|_{c^{2}}\right) \min \left\{1, t^{-1}\right\} \\
& \leqq C\left(\left\|B^{0}\right\|_{c^{2}}+\left\|\int \sum_{\alpha} e_{\alpha} m_{\alpha}^{3} g_{\alpha}^{0} \hat{u} d u\right\|_{c^{1}}+\left\|E^{0}\right\|_{c^{2}}\right)(1+t)^{-1} \\
& \leqq C \varepsilon(1+t)^{-1} .
\end{aligned}
$$

Again using (18) we have by (7),

$$
\begin{aligned}
\left|E_{z}-\mathscr{E}\right| & \leqq t^{-1} \int_{|x-y|=t} \int_{|u|<C} C\left|\sum_{\alpha} e_{\alpha} m_{\alpha}^{3} g_{\alpha}^{0}\right| d u d S_{y} \\
& \leqq t^{-1} C\left\|\int_{|u|<C} C\left|\sum_{\alpha} e_{\alpha} m_{\alpha}^{3} g_{\alpha}^{0}\right| d u\right\|_{c^{0}} \min \left\{t^{2}, 1\right\} \\
& \leqq C \varepsilon(1+t)^{-1}
\end{aligned}
$$


and

$$
\begin{aligned}
& \left|\nabla_{x}\left(E_{z}-\mathscr{E}\right)\right| \\
& \quad=t\left|\nabla_{x}\left(\int_{|w|=1} \int(1+\hat{u} \cdot w)^{-1}(w-(\hat{u} \cdot w) \hat{u})\left(\sum_{\alpha} e_{\alpha} m_{\alpha}^{3} g_{\alpha}^{0}(x+t w, u)\right) d u d w\right)\right| \\
& \quad=t^{-1}\left|\int_{|x-y|=t} \int(1+\hat{u} \cdot w)^{-1}(w-(\hat{u} \cdot w) \hat{u}) \nabla_{x}\left(\sum_{\alpha} e_{\alpha} m_{\alpha}^{3} g_{\alpha}^{0}\right)\right|_{(y, u)} d u d S_{y} \mid \\
& \quad \leqq t^{-1} C\left\|\int \sum_{\alpha} e_{\alpha} m_{\alpha}^{3} g_{\alpha}^{0} d u\right\|_{c^{1}} \min \left\{t^{2}, 1\right\} \leqq C \varepsilon(1+t)^{-1} .
\end{aligned}
$$

We may estimate $A_{z}-\partial E_{z} / \partial x_{k}$ in a highly similar manner, when we observe that

$$
\left|S g_{\alpha}^{0}(y, u)\right|=\left|e_{\alpha} m_{\alpha}^{-1} K(0, y) \cdot \nabla_{u} g_{\alpha}^{0}(y, u)\right| \leqq C \varepsilon .
$$

The result is, again,

$$
\left|A_{z}-\frac{\partial E_{z}}{\partial x_{k}}\right| \leqq C \varepsilon(1+t)^{-1} .
$$

Now, collecting (20) through (23) we get

$$
\left|E_{z}\right|+\left|A_{z}\right| \leqq C \varepsilon(1+t)^{-1}
$$

However, $E_{z}$ and $A_{z}$ vanish on the sets $|x|<t-C$ and $|x|>t+C$, so

$$
\left|E_{z}\right|+\left|A_{z}\right| \leqq C \varepsilon(1+k+t+|x|)^{-1}(1+k+t-|x|)^{-2} \mathscr{X}(t, x) .
$$

The estimation of $\left|B_{z}\right|+\left|\tilde{A}_{z}\right|$ is very similar and is omitted.

\section{Estimation Using Neutrality}

As noted earlier if we take data in $\mathscr{D}(k, M, \varepsilon)$ with $\varepsilon<\eta=\eta_{2}$, then it follows from the continuation result of [4] that a unique continuously differentiable solution exists and satisfies the (FSC) on some time interval $\left[0, T_{\varepsilon}\right.$ ). We may take $T_{\varepsilon}$ to be as large as possible. In this section we make crude estimates using the neutrality assumption, i.e.

$$
\left\|\sum_{\alpha} e_{\alpha} m_{\alpha}^{3} g_{\alpha}^{0}\right\|_{c^{1}}+\left\|E^{0}\right\|_{c^{2}}+\left\|B^{0}\right\|_{c^{2}}<\varepsilon,
$$

which show that $T_{\varepsilon}$ tends to infinity as $\varepsilon \rightarrow 0$. Although $T_{\varepsilon}$ actually depends on the data, the following estimates depend only $\varepsilon$ (and $k, M, m_{\alpha}, e_{\alpha}$ ).

Lemma 5. Let us denote

$$
\mathscr{V}_{a}(s)=\mathscr{V}_{\alpha}\left(s, t, x, m_{\alpha} u\right)
$$

and

$$
\mathscr{X}_{\alpha}(s)=\mathscr{X}_{\alpha}\left(s, t, x, m_{\alpha} u\right)
$$


Then for all $(t, x, u) \in\left[0, T_{\varepsilon}\right) \times \mathbb{R}^{3} \times \mathbb{R}^{3}$, we have

$$
\begin{aligned}
& \left|m_{\alpha}^{-1} \mathscr{V}_{\alpha}(0)-m_{1}^{-1} \mathscr{V}_{1}(0)\right|+\left|\mathscr{X}_{\alpha}(0)-\mathscr{X}_{1}(0)\right| \\
& \quad \leqq C \int_{0}^{t}(1+s)\left(\|E(s)\|_{c^{0}}+\|B(s)\|_{c^{0}}\right) d s \\
& \left|\nabla_{(x, u)}\left(m_{\alpha}^{-1} \mathscr{V}_{\alpha}(0)-m_{1}^{-1} \mathscr{V}_{1}(0)\right)\right|+\left|\nabla_{(x, u)}\left(\mathscr{X}_{\alpha}(0)-\mathscr{X}_{1}(0)\right)\right| \\
& \quad \leqq C(t) \int_{0}^{t}\left(\|E(s)\|_{c^{1}}+\|B(s)\|_{c^{1}}\right) d s
\end{aligned}
$$

and

$$
\left|\nabla_{(x, u)} \mathscr{X}_{\alpha}(0)\right|+\left|\nabla_{(x, u)} \mathscr{V}_{\alpha}(0)\right| \leqq C(t)
$$

Proof. Recall that

and

$$
\dot{\mathscr{X}}_{\alpha}(\tau)=\widehat{\mathscr{V}}_{\alpha}(\tau)=\left(m_{\alpha}^{2}+\left|\mathscr{V}_{\alpha}(\tau)\right|^{2}\right)^{-1 / 2} \mathscr{V}_{\alpha}(\tau)
$$

$$
\dot{\mathscr{V}}_{\alpha}(\tau)=e_{\alpha}\left[E\left(\tau, \mathscr{X}_{\alpha}(\tau)\right)+\widehat{\mathscr{V}}_{\alpha}(\tau)_{\Lambda} B\left(\tau, \mathscr{X}_{\alpha}(\tau)\right)\right]
$$

Note that

$$
\begin{aligned}
\left|\nabla_{(x, u)} \dot{\mathscr{V}}_{\alpha}(\tau)\right| \leqq & C\left[\|E(\tau)\|_{c^{1}}\left|\nabla_{(x, u)} \mathscr{X}_{\alpha}(\tau)\right|\right. \\
& \left.+\left|\nabla_{(x, u)} \hat{\mathscr{V}}_{\alpha}(\tau)\right|\|B(\tau)\|_{c^{0}}+\|B(\tau)\|_{c^{1}}\left|\nabla_{(x, u)} \mathscr{X}_{\alpha}(\tau)\right|\right] \\
\leqq & C\left(\|E(\tau)\|_{c^{1}}+\|B(\tau)\|_{c^{1}}\right)\left(\left|\nabla_{(x, u)} \mathscr{X}_{\alpha}(\tau)\right|+\left|\nabla_{(x, u)} \mathscr{V}_{\alpha}(\tau)\right|\right)
\end{aligned}
$$

and

$$
\left|\nabla_{(x, u)} \dot{\mathscr{X}}_{\alpha}(\tau)\right|=\left|\nabla_{(x, u)} \widehat{\mathscr{V}}_{\alpha}(\tau)\right| \leqq C\left|\nabla_{(x, u)} \mathscr{V}_{\alpha}(\tau)\right| .
$$

Now for $0 \leqq s \leqq \tau \leqq t<T_{\varepsilon}$ we use (25), (26) and the (FSC) to obtain

$$
\begin{aligned}
& \left|\nabla_{(x, u)} \mathscr{X}_{\alpha}(s)\right|+\left|\nabla_{(x, u)} \mathscr{V}_{\alpha}(s)\right| \\
& \quad \leqq C+C \int_{s}^{t}\left(1+|| E(\tau)\left\|_{c^{1}}+|| B(\tau)\right\|_{c^{1}}\right)\left(\left|\nabla_{(x, u)} \mathscr{X}_{\alpha}(\tau)\right|+\left|\nabla_{(x, u)} \mathscr{V}_{\alpha}(\tau)\right|\right) d \tau \\
& \quad \leqq C+C \int_{s}^{t}\left(\left|\nabla_{(x, u)} \mathscr{X}_{\alpha}(\tau)\right|+\left|\nabla_{(x, u)} \mathscr{V}_{\alpha}(\tau)\right|\right) d \tau .
\end{aligned}
$$

Hence by Gronwall's inequality we have

$$
\left|\nabla_{(x, u)} \mathscr{X}_{\alpha}(s)\right|+\left|\nabla_{(x, u)} \mathscr{V}_{\alpha}(s)\right| \leqq C(t)
$$

for all $s \in[0, t]$.

Note that $\mathscr{X}_{\alpha}(s)-\mathscr{X}_{1}(s), \nabla_{(x, u)}\left(\mathscr{X}_{\alpha}(s)-\mathscr{X}_{1}(s)\right), m_{\alpha}^{-1} \mathscr{V}_{\alpha}(s)-m_{1}^{-1} \mathscr{V}_{1}(s)$, and $\nabla_{(x, u)}\left(m_{\alpha}^{-1} \mathscr{V}_{\alpha}(s)-m_{1}^{-1} \mathscr{V}_{1}(s)\right)$ all vanish when $s=t$. Thus for $0<s<t<T_{\varepsilon}$,

$$
\begin{aligned}
\left|m_{\alpha}^{-1} \mathscr{V}_{\alpha}(s)-m_{1}^{-1} \mathscr{V}_{1}(s)\right| & \leqq \int_{s}^{t}\left(\left|m_{\alpha}^{-1} \dot{\mathscr{V}}_{\alpha}(\tau)\right|+\left|m_{1}^{-1} \dot{\mathscr{V}}_{1}(\tau)\right|\right) d \tau \\
& \leqq C \int_{s}^{t}\left(\|E(\tau)\|_{c^{0}}+\|B(\tau)\|_{c^{0}}\right) d \tau
\end{aligned}
$$


and by (25) and (27)

$$
\left|\nabla_{(x, u)}\left(m_{\alpha}^{-1} \mathscr{V}_{\alpha}(s)-m_{1}^{-1} \mathscr{V}_{1}(s)\right)\right| \leqq C(t) \int_{s}^{t}\left(\|E(\tau)\|_{c^{1}}+\|B(\tau)\|_{c^{1}}\right) d \tau
$$

In the same vein, by (28) we have

$$
\begin{aligned}
& \left|\mathscr{X}_{\alpha}(s)-\mathscr{X}_{1}(s)\right| \leqq \int_{s}^{t}\left|\hat{\mathscr{V}}_{\alpha}(\tau)-\hat{\mathscr{V}}_{1}(\tau)\right| d \tau \\
& \quad=\int_{s}^{t}\left|\frac{m_{\alpha}^{-1} \mathscr{V}_{\alpha}}{\sqrt{1+\left|m_{\alpha}^{-1} \mathscr{V}_{\alpha}\right|^{2}}}-\frac{m_{1}^{-1} \mathscr{V}_{1}}{\sqrt{1+\left|m_{1}^{-1} \mathscr{V}_{1}\right|^{2}}}\right| d \tau \\
& \quad \leqq C \int_{s}^{t}\left|m_{\alpha}^{-1} \mathscr{V}_{\alpha}-m_{1}^{-1} \mathscr{V}_{1}\right| d \tau \leqq C \int_{s}^{t} \int_{\tau}^{t} C\left(\|E(\bar{\tau})\|_{c^{0}}+\|B(\bar{\tau})\|_{c^{0}}\right) d \bar{\tau} d \tau \\
& \quad=C \int_{s}^{t}(\bar{\tau}-s)\left(\|E(\bar{\tau})\|_{c^{0}}+\|B(\bar{\tau})\|_{c^{0}}\right) d \bar{\tau} .
\end{aligned}
$$

Let " $\partial$ " denote partial derivative with respect to any component of $x$ or $u$. We compute

$$
\begin{aligned}
\partial\left(\hat{\mathscr{V}}_{\alpha}^{k}-\hat{\mathscr{V}}_{1}^{k}\right)= & -\left(m_{\alpha}^{2}+\left|\mathscr{V}_{\alpha}\right|^{2}\right)^{-3 / 2} \mathscr{V}_{\alpha}^{k} \mathscr{V}_{\alpha} \cdot \partial \mathscr{V}_{\alpha}+\left(m_{\alpha}^{2}+\left|\mathscr{V}_{\alpha}\right|^{2}\right)^{-1 / 2} \partial \mathscr{V}_{\alpha}^{k} \\
& +\left(m_{1}^{2}+\left|\mathscr{V}_{1}\right|^{2}\right)^{-3 / 2} \mathscr{V}_{1}^{k} \mathscr{V}_{1} \cdot \partial \mathscr{V}_{1}-\left(m_{1}^{2}+\left|\mathscr{V}_{1}\right|^{2}\right)^{-1 / 2} \partial \mathscr{V}_{\alpha}^{k}
\end{aligned}
$$

Define for $u \in \mathbb{R}^{3}$

and

$$
\beta(u)=-\left(1+|u|^{2}\right)^{-3 / 2} u^{k} u
$$

$$
\xi(u)=\left(1+|u|^{2}\right)^{-1 / 2} .
$$

Then by (27), (28), and (29),

$$
\begin{aligned}
\left|\partial\left(\hat{\mathscr{V}}_{\alpha}^{k}(s)-\hat{\mathscr{V}}_{1}^{k}(s)\right)\right|= & \mid \beta\left(m_{\alpha}^{-1} \mathscr{V}_{\alpha}\right) \cdot \partial\left(m_{\alpha}^{-1} \mathscr{V}_{\alpha}\right)-\beta\left(m_{1}^{-1} \mathscr{V}_{1}\right) \cdot \partial\left(m_{1}^{-1} \mathscr{V}_{1}\right) \\
& +\xi\left(m_{\alpha}^{-1} \mathscr{V}_{\alpha}\right) \partial\left(m_{\alpha}^{-1} \mathscr{V}_{\alpha}^{k}\right)-\xi\left(m_{1}^{-1} \mathscr{V}_{1}\right) \partial\left(m_{1}^{-1} \mathscr{V}_{1}^{k}\right) \mid \\
\leqq & \left|\beta\left(m_{\alpha}^{-1} \mathscr{V}_{\alpha}\right)\right|\left|\partial\left(m_{\alpha}^{-1} \mathscr{V}_{\alpha}-m_{1}^{-1} \mathscr{V}_{1}\right)\right| \\
& +\left|\beta\left(m_{\alpha}^{-1} \mathscr{V}_{\alpha}\right)-\beta\left(m_{1}^{-1} \mathscr{V}_{1}\right)\right|\left|\partial\left(m_{1}^{-1} \mathscr{V}_{1}\right)\right| \\
& +\left|\xi\left(m_{\alpha}^{-1} \mathscr{V}_{\alpha}\right)\right|\left|\partial\left(m_{\alpha}^{-1} \mathscr{V}_{\alpha}^{k}-m_{1}^{-1} \mathscr{V}_{1}^{k}\right)\right| \\
& +\left|\xi\left(m_{\alpha}^{-1} \mathscr{V}_{\alpha}\right)-\xi\left(m_{1}^{-1} \mathscr{V}_{1}\right)\right|\left|\partial\left(m_{1}^{-1} \mathscr{V}_{1}^{k}\right)\right| \\
\leqq & C\left|\partial\left(m_{\alpha}^{-1} \mathscr{V}_{\alpha}-m_{1}^{-1} \mathscr{V}_{1}\right)\right| \\
& +C\left|m_{\alpha}^{-1} \mathscr{V}_{\alpha}-m_{1}^{-1} \mathscr{V}_{1} \| \partial \mathscr{V}_{1}\right| \\
\leq & C(t) \int_{s}^{t}\left(\|E(\tau)\|_{c^{1}}+\|B(\tau)\|_{c^{1}}\right) d \tau
\end{aligned}
$$

Thus

$$
\begin{aligned}
\left|\nabla_{(x, u)}\left(\mathscr{X}_{\alpha}(s)-\mathscr{X}_{1}(s)\right)\right| & \leqq \int_{s}^{t}\left|\nabla_{(x, u)}\left(\hat{\mathscr{V}}_{\alpha}(\tau)-\hat{\mathscr{V}}_{1}(\tau)\right)\right| d \tau \\
& \leqq \int_{s}^{t} C(t) \int_{\tau}^{t}\left(\|E(\bar{\tau})\|_{c^{1}}+\|B(\bar{\tau})\|_{c^{1}}\right) d \bar{\tau} d \tau \\
& \leqq C(t) \int_{s}^{t}\left(\|E(\tau)\|_{\mathcal{c}^{1}}+\|B(\tau)\|_{c^{1}}\right) d \tau .
\end{aligned}
$$

The proof is now complete. 
Lemma 6. For $0 \leqq t<T_{\varepsilon}$,

and

$$
\left\|\sum_{\alpha} e_{\alpha} m_{\alpha}^{3} g_{\alpha}(t)\right\|_{c^{0}} \leqq \varepsilon+C \int_{0}^{t}(1+s)\left(\|E(s)\|_{c^{0}}+\|B(s)\|_{c^{0}}\right) d s,
$$

$$
\left\|\sum_{\alpha} e_{\alpha} m_{\alpha}^{3} g_{\alpha}(t)\right\|_{c^{1}} \leqq C(t) \varepsilon+C(t) \int_{0}^{t}\left(\|E(s)\|_{c^{1}}+\|B(s)\|_{c^{1}}\right) d s .
$$

Proof. First we recall that

$$
g_{\alpha}(t, x, u)=g_{\alpha}^{0}\left(\mathscr{X}_{\alpha}\left(0, t, x, m_{\alpha} u\right), m_{\alpha}^{-1} \mathscr{V}_{\alpha}\left(0, t, x, m_{\alpha} u\right)\right),
$$

so we may write

$$
\begin{aligned}
\sum_{\alpha} e_{\alpha} m_{\alpha}^{3} g_{\alpha}(t, x, u)= & \sum_{\alpha} e_{\alpha} m_{\alpha}^{3}\left[g_{\alpha}^{0}\left(\mathscr{X}_{\alpha}\left(0, t, x, m_{\alpha} u\right), m_{\alpha}^{-1} \mathscr{V}_{\alpha}\left(0, t, x, m_{\alpha} u\right)\right),\right. \\
& \left.-g_{\alpha}^{0}\left(\mathscr{X}_{1}\left(0, t, x, m_{1} u\right), m_{1}^{-1} \mathscr{V}_{1}\left(0, t, x, m_{1} u\right)\right)\right] \\
& +\sum_{\alpha} e_{\alpha} m_{\alpha}^{3} g_{\alpha}^{0}\left(\mathscr{X}_{1}\left(0, t, x, m_{1} u\right), m_{1}^{-1} \mathscr{V}_{1}\left(0, t, x, m_{1} u\right)\right) .
\end{aligned}
$$

Now by (30), Lemma 5 , and the neutrality assumption

$$
\begin{aligned}
\left|\sum_{\alpha} e_{\alpha} m_{\alpha}^{3} g_{\alpha}(t, x, u)\right| & \leqq \sum_{\alpha}\left|e_{\alpha}\right| m_{\alpha}^{3}\left\|g_{\alpha}^{0}\right\|_{c^{1}}\left(\left|\mathscr{X}_{\alpha}(0)-\mathscr{X}_{1}(0)\right|\right. \\
& \left.+\left|m_{\alpha}^{-1} \mathscr{V}_{\alpha}(0)-m_{1}^{-1} \mathscr{V}_{1}(0)\right|\right)+\left\|\sum_{\alpha} e_{\alpha} m_{\alpha}^{3} g_{\alpha}^{0}\right\|_{c^{0}} \\
& \leqq \varepsilon+C \int_{0}^{t}(1+s)\left(\|E(s)\|_{c^{0}}+\|B(s)\|_{c^{0}}\right) d s .
\end{aligned}
$$

Let " $\partial$ " denote partial derivative with respect to any component of $x$ or $u$. Then again using (30), Lemma 5, and the neutrality assumption we have

$$
\begin{aligned}
& \left|\partial\left(\sum_{\alpha} e_{\alpha} m_{\alpha}^{3} g_{\alpha}(t, x, u)\right)\right| \leqq \mid \sum_{\alpha} e_{\alpha} m_{\alpha}^{3}\left[\nabla_{x} g_{\alpha}^{0}\left(\mathscr{X}_{\alpha}(0), m_{\alpha}^{-1} \mathscr{V}_{\alpha}(0)\right) \cdot \partial\left(\mathscr{X}_{\alpha}(0)\right)\right. \\
& +\nabla_{u} g_{\alpha}^{0}\left(\mathscr{X}_{\alpha}(0), m_{\alpha}^{-1} \mathscr{V}_{\alpha}(0)\right) \cdot \partial\left(m_{\alpha}^{-1} \mathscr{V}_{\alpha}(0)\right)-\nabla_{x} g_{\alpha}^{0}\left(\mathscr{X}_{1}(0), m_{1}^{-1} \mathscr{V}_{1}(0)\right) \cdot \partial\left(\mathscr{X}_{1}(0)\right) \\
& \left.-\nabla_{u} g_{\alpha}^{0}\left(\mathscr{X}_{1}(0), m_{1}^{-1} \mathscr{V}_{1}(0)\right) \cdot \partial\left(m_{1}^{-1} \mathscr{V}_{1}(0)\right)\right] \\
& +\mid \sum_{\alpha} e_{\alpha} m_{\alpha}^{3}\left[\nabla_{x} g_{\alpha}^{0}\left(\mathscr{X}_{1}(0), m_{1}^{-1} \mathscr{V}_{1}(0)\right) \cdot \partial\left(\mathscr{X}_{1}(0)\right)\right. \\
& \left.+\nabla_{u} g_{\alpha}^{0}\left(\mathscr{X}_{1}(0), m_{1}^{-1} \mathscr{V}_{1}(0)\right) \cdot \partial\left(m_{1}^{-1} \mathscr{V}_{1}(0)\right)\right] \\
& \leqq C \sum_{\alpha}\left(\left\|g_{\alpha}^{0}\right\|_{c^{1}}\left[\left|\partial\left(\mathscr{X}_{\alpha}(0)-\mathscr{X}_{1}(0)\right)\right|+\left|\partial\left(m_{\alpha}^{-1} \mathscr{V}_{\alpha}(0)-m_{1}^{-1} \mathscr{V}_{1}(0)\right)\right|\right]\right. \\
& +\left|\nabla_{x} g_{\alpha}^{0}\left(\mathscr{X}_{\alpha}(0), m_{\alpha}^{-1} \mathscr{V}_{\alpha}(0)\right)-\nabla_{x} g_{\alpha}^{0}\left(\mathscr{X}_{1}(0), m_{1}^{-1} \mathscr{V}_{1}(0)\right)\right|\left|\partial\left(\mathscr{X}_{1}(0)\right)\right| \\
& \left.+\left|\nabla_{u} g_{\alpha}^{0}\left(\mathscr{X}_{\alpha}(0), m_{\alpha}^{-1} \mathscr{V}_{\alpha}(0)\right)-\nabla_{u} g_{\alpha}^{0}\left(\mathscr{X}_{1}(0), m_{1}^{-1} \mathscr{V}_{1}(0)\right) \| \partial\left(m_{1}^{-1} \mathscr{V}_{1}(0)\right)\right|\right) \\
& +\left\|\sum_{\alpha} e_{\alpha} m_{\alpha}^{3} g_{\alpha}^{0}\right\|_{c^{1}}\left(\left|\partial\left(\mathscr{X}_{1}(0)\right)\right|+\left|\partial\left(m_{1}^{-1} \mathscr{V}_{1}(0)\right)\right|\right)
\end{aligned}
$$




$$
\begin{aligned}
\leqq & C(t)\left(\sum_{\alpha}\left\|g_{\alpha}^{0}\right\|_{c^{1}}\right) \int_{0}^{t}\left(\|E(s)\|_{c^{1}}+\|B(s)\|_{c^{1}}\right) d s \\
& +C(t) \sum_{\alpha}\left\|g_{\alpha}^{0}\right\|_{c^{2}}\left(\left|\mathscr{X}_{\alpha}(0)-\mathscr{X}_{1}(0)\right|+\left|m_{\alpha}^{-1} \mathscr{V}_{\alpha}(0)-m_{1}^{-1} \mathscr{V}_{1}(0)\right|\right)+C(t) \varepsilon \\
\leqq & C(t) \varepsilon+C(t) \int_{0}^{t}\left(\|E(s)\|_{c^{1}}+\|B(s)\|_{c^{1}}\right) d s .
\end{aligned}
$$

The lemma now follows.

Lemma 7. For $t \in\left[0, T_{\varepsilon}\right)$,

$$
\begin{aligned}
& \|E(t)\|_{c^{1}}+\|B(t)\|_{c^{1}}+\left\|E_{T}(t)\right\|_{c^{0}}+\left\|B_{T}(t)\right\|_{c^{0}}+\left\|A_{T T}(t)\right\|_{c^{0}}+\left\|\tilde{A}_{T T}(t)\right\|_{c^{0}}+\| A_{S T}(t) \\
& \quad+A_{T S}(t)\left\|_{c^{0}}+\right\| \tilde{A}_{S T}(t)+\tilde{A}_{T S}(t)\left\|_{c^{0}}+\right\| A_{w}(t)\left\|_{c^{0}}+\right\| \tilde{A}_{w}(t) \|_{c^{0}} \leqq \varepsilon C(t) .
\end{aligned}
$$

We point out that it follows from Lemma 7 that $T_{\varepsilon}$ tends to infinity as $\varepsilon \rightarrow 0$. Proof. Since $t<T_{\varepsilon}$, the (FSC) holds and hence by Lemma 1 the support of

$$
u \mapsto \sum_{\alpha} e_{\alpha} m_{\alpha}^{3} g_{\alpha}(t, x, u)
$$

is bounded. Hence by (8) and Lemma 6

$$
\begin{aligned}
\left|E_{T}\right| \leqq & \int_{|x-y|<t}|x-y|^{-2} \int_{|u|<C}\left\|C \sum_{\alpha} e_{\alpha} m_{\alpha}^{3} g_{\alpha}(t-|x-y|)\right\|_{c^{0}} d u d y \\
\leqq & C \int_{|x-y|<t}|x-y|^{-2}[C(t-|x-y|) \varepsilon \\
& \left.+C(t-|x-y|) \int_{0}^{t-|x-y|}\left(\|E(s)\|_{c^{1}}+\|B(s)\|_{c^{1}}\right) d s\right] d y \\
\leqq & C(t) \int_{|x-y|<t}|x-y|^{-2}\left(\varepsilon+\int_{0}^{t}\left[\|E(s)\|_{c^{1}}+\|B(s)\|_{c^{1}}\right] d s\right) d y \\
\leqq & C(t)\left(\varepsilon+\int_{0}^{t}\left[\|E(s)\|_{c^{1}}+\|B(s)\|_{c^{1}}\right] d s\right) .
\end{aligned}
$$

Similarly by (9)

$$
\begin{aligned}
\left|E_{S}\right| \leqq & \int_{|x-y|<t}|x-y|^{-1} \int_{|u|<C} C\left\|\sum_{\alpha} e_{\alpha}^{2} m_{\alpha}^{2} g_{\alpha}(t-|x-y|)\right\|_{c^{0}} \\
& \cdot\left(\|E(t-|x-y|)\|_{c^{0}}+\|B(t-|x-y|)\|_{c^{0}}\right) d u d y \\
\leqq & C \int_{|x-y|<t}|x-y|^{-1}\left(\|E(t-|x-y|)\|_{c^{0}}+\|B(t-|x-y|)\|_{c^{0}}\right) d y \\
\leqq & C(t) \int_{0}^{t}\left(\|E(s)\|_{c^{1}}+\|B(s)\|_{c_{1}}\right) d s .
\end{aligned}
$$

Also using the boundedness of the $u$-support we have by (16) and Lemma 6

$$
\left|A_{w}\right| \leqq \int_{|u|<C} C\left\|\sum_{\alpha} e_{\alpha} m_{\alpha}^{3} g_{\alpha}(t)\right\|_{c^{0}} d u \leqq C(t)\left(\varepsilon+\int_{0}^{t}\left(\|E(s)\|_{c^{1}}+\|B(s)\|_{c^{1}}\right) d s\right) .
$$


It follows from Lemma 4 that

$$
\left|E_{Z}\right|+\left|A_{z}\right| \leqq C \varepsilon .
$$

Now we consider $A_{T T}, A_{S T}+A_{T S}$, and $A_{S S}$. In light of (14) we may use (11) to write

$$
\begin{aligned}
&\left|A_{T T}\right|=\left|\int_{|x-y|<t}\right| x-\left.y\right|^{-3} \int_{|u|<C} a(w, \hat{u})\left(\sum_{\alpha} e_{\alpha} m_{\alpha}^{3} g_{\alpha}(t-|x-y|, y, u)\right. \\
&\left.-\sum_{\alpha} e_{\alpha} m_{\alpha}^{3} g_{\alpha}(t-|x-y|, x, u)\right) d u d y \mid \\
& \leqq \\
& \int_{|x-y|<t}|x-y|^{-3} \int_{|u|<C} C\left\|\sum_{\alpha} e_{\alpha} m_{\alpha}^{3} g_{\alpha}(t-|x-y|)\right\|_{c^{1}}|x-y| d u d y \\
& \leqq \int_{|x-y|<t}|x-y|^{-2} C\left\|\sum_{\alpha} e_{\alpha} m_{\alpha}^{3} g_{\alpha}(t-|x-y|)\right\|_{c^{1}} d y .
\end{aligned}
$$

Now by Lemma 6

$$
\begin{aligned}
\left|A_{T T}\right| & \leqq C \int_{|x-y|<t}|x-y|^{-2} C(t-|x-y|)\left[\varepsilon+\int_{0}^{t-|x-y|}\left(\|E(s)\|_{c^{1}}+\|B(s)\|_{c^{1}}\right) d s\right] d y \\
& \leqq C(t)\left[\varepsilon+\int_{0}^{t}\left(\|E(s)\|_{c^{1}}+\|B(s)\|_{c^{1}}\right) d s\right] .
\end{aligned}
$$

To estimate $A_{T S}+A_{S T}$ we integrate by parts (in the $u$ integral) in (12) and use the identity $S g_{\alpha}=-\nabla_{u} \cdot\left(e_{\alpha} m_{\alpha}^{-1} K g_{\alpha}\right)$ :

$$
\begin{aligned}
\left|A_{T S}+A_{S T}\right| & =\left|\int_{|x-y|<t}\right| x-\left.\left.y\right|^{-2} \int_{|u|<C} \nabla_{u}[b(w, \hat{u})] \cdot\left(\sum_{\alpha} e_{\alpha}^{2} m_{\alpha}^{2} g_{\alpha} K\right)\right|_{(t-|x-y|, y, u)} d u d y \mid \\
& \leqq \int_{|x-y|<t}|x-y|^{-2} C\left[\|E(t-|x-y|)\|_{c^{0}}+\|B(t-|x-y|)\|_{c^{0}}\right] d y \\
& =C \int_{0}^{t}\left(\|E(s)\|_{c^{1}}+\|B(s)\|_{c^{1}}\right) d s
\end{aligned}
$$

In order to perform a similar integration by parts in (13), note that

$$
\begin{aligned}
S^{2} g_{\alpha} & =S\left(-\nabla_{u} \cdot\left(e_{\alpha} m_{\alpha}^{-1} g_{\alpha} K\right)\right) \\
& =-\nabla_{u} \cdot\left(S\left(e_{\alpha} m_{\alpha}^{-1} g_{\alpha} K\right)\right)+\sum_{i, j} \frac{\partial \hat{u}_{j}}{\partial u_{i}} \frac{\partial\left(e_{\alpha} m_{\alpha}^{-1} g_{\alpha} K_{i}\right)}{\partial x_{j}},
\end{aligned}
$$

and hence

$$
\begin{aligned}
A_{S S}= & \left.\int_{|x-y|<t}|x-y|^{-1} \int_{|u|<C} \nabla_{u}(c(w, \hat{u})) \cdot\left(\sum_{\alpha} e_{\alpha}^{2} m_{\alpha}^{2} S\left(g_{\alpha} K\right)\right)\right|_{(t-|x-y|, y, u)} d u d y \\
& +\left.\int_{|x-y|<t}|x-y|^{-1} \int_{|u|<C} c(w, \hat{u}) \sum_{\alpha} e_{\alpha}^{2} m_{\alpha}^{2} \sum_{i, j} \frac{\partial \hat{u}_{j}}{\partial u_{i}} \frac{\partial\left(g_{\alpha} K_{i}\right)}{\partial y_{j}}\right|_{(t-|x-y|, y, u)} d u d y .
\end{aligned}
$$


Note also that

so

$$
S\left(g_{\alpha} K\right)=\left(S g_{\alpha}\right) K+g_{\alpha}(S K)=-\left(\nabla_{u} \cdot\left(e_{\alpha} m_{\alpha}^{-1} K g_{\alpha}\right) K\right)+g_{\alpha}(S K),
$$

where

$$
A_{S S}=\mathrm{I}+\mathrm{II}+\mathrm{III}
$$

$$
\begin{gathered}
\mathrm{I}=\left.\int_{|x-y|<t}|x-y|^{-1} \int_{|u|<C} \sum_{i, j} \frac{\partial^{2}(c(w, \hat{u}))}{\partial u_{i} \partial u_{j}} \sum_{\alpha} e_{\alpha}^{3} m_{\alpha} g_{\alpha} K_{i} K_{j}\right|_{(t-|x-y|, y, u)} d u d y, \\
\mathrm{II}=\left.\int_{|x-y|<t}|x-y|^{-1} \int_{|u|<C} \nabla_{u}(c(w, \hat{u})) \cdot\left(\sum_{\alpha} e_{\alpha}^{2} m_{\alpha}^{2} g_{\alpha} S K\right)\right|_{(t-|x-y|, y, u)} d u d y, \\
\mathrm{III}=\left.\int_{|x-y|<t}|x-y|^{-1} \int_{|u|<C} c(w, \hat{u}) \sum_{\alpha} e_{\alpha}^{2} m_{\alpha}^{2} \sum_{i, j} \frac{\partial \hat{u}_{j}}{\partial u_{i}} \frac{\partial}{\partial y_{j}}\left(g_{\alpha} K_{i}\right)\right|_{(t-|x-y|, y, u)} d u d y .
\end{gathered}
$$

Using the (FSC) and the bounded $u$ support, we have

$$
|I| \leqq \int_{|x-y|<t}|x-y|^{-1} C\|K(t-|x-y|)\|_{c^{0}}^{2} d y \leqq C(t) \int_{0}^{t}\left(\|E(s)\|_{c^{0}}+\|B(s)\|_{c^{0}}\right) d s .
$$

Next note that

$\left\|g_{\alpha}(S K)(t)\right\|_{c^{0}}+\left\|\nabla_{y}\left(g_{\alpha} K\right)(t)\right\|_{c^{0}} \leqq C\left(\|K(t)\|_{c^{1}}+\left\|\partial_{t} K(t)\right\|_{c^{0}}\right)+\|K(t)\|_{c^{0}}\left\|g_{\alpha}(t)\right\|_{c^{1}}$.

By Lemma 6

$$
|j(t, y)| \leqq \int_{|u|<C}\left|\sum_{\alpha} e_{\alpha} m_{\alpha}^{3} g_{\alpha}(t, y, u)\right| d u \leqq C(t) \varepsilon+C(t) \int_{0}^{t}\left(\|E(s)\|_{c^{1}}+\|B(s)\|_{c^{1}}\right) d s,
$$

so by Maxwell's equations

$$
\left\|\partial_{t} K(t)\right\|_{c^{0}} \leqq\|K(t)\|_{c^{1}}+C(t) \varepsilon+C(t) \int_{0}^{t}\left(\|E(s)\|_{c^{1}}+\|B(s)\|_{c^{1}}\right) d s .
$$

By Lemma 5

$$
\begin{aligned}
\left|\nabla_{x} g_{\alpha}(t, y, u)\right| & =\left|\nabla_{x}\left[g_{\alpha}^{0}\left(\mathscr{X}_{\alpha}\left(0, t, x, m_{\alpha} u\right), m_{\alpha}^{-1} \mathscr{V}_{\alpha}\left(0, t, x, m_{\alpha} u\right)\right)\right]\right| \\
& \leqq\left\|\nabla_{x} g_{\alpha}^{0}\right\|_{c^{0}}\left|\nabla_{x} \mathscr{X}_{\alpha}\left(0, t, x, m_{\alpha} u\right)\right|+\left\|\nabla_{u} g_{\alpha}^{0}\right\|_{c^{0}}\left|\nabla_{x} \mathscr{V}_{\alpha}\left(0, t, x, m_{\alpha} u\right)\right| m_{\alpha}^{-1} \\
& \leqq C(t)
\end{aligned}
$$

Similarly

so

$$
\left|\nabla_{u} g_{\alpha}(t, y, u)\right| \leqq C(t)
$$

$$
\left\|g_{\alpha}(t)\right\|_{c^{1}} \leqq C(t) .
$$

Now use of (40) and (41) in (39) yields

$$
\left\|g_{\alpha}(S K)(t)\right\|_{c^{0}}+\left\|\nabla_{y}\left(g_{\alpha} K\right)(t)\right\|_{c^{0}} \leqq C(t)\left[\|K(t)\|_{c^{1}}+\varepsilon+\int_{0}^{t}\left(\|E(s)\|_{c^{1}}+\|B(s)\|_{c^{1}}\right) d s\right],
$$


and hence

$$
\begin{aligned}
|\mathrm{II}|+|\mathrm{III}| \leqq & \int_{|x-y|<t}|x-y|^{-1} C(t) \\
& \cdot\left[\|K(t-|x-y|)\|_{c^{1}}+\varepsilon+\int_{0}^{t-|x-y|}\left(\|E(s)\|_{c^{1}}+\|B(s)\|_{c^{1}}\right) d s\right] d y \\
\leqq & C(t)\left[\varepsilon+\int_{0}^{t}\left(\|E(s)\|_{c^{1}}+\|B(s)\|_{c^{1}}\right) d s\right] .
\end{aligned}
$$

Therefore, by (37), (38) and (42)

$$
\left|A_{S S}\right| \leqq C(t)\left(\varepsilon+\int_{0}^{t}\left[\|E(s)\|_{c^{1}}+\|B(s)\|_{c^{1}}\right] d s\right) .
$$

Now collecting the estimates (31) through (36) and (43) we have (by (6) and (10))

$$
\|E(t)\|_{c^{1}} \leqq C(t)\left(\varepsilon+\int_{0}^{t}\left[\|E(s)\|_{c^{1}}+\|B(s)\|_{c^{1}}\right] d s\right) .
$$

The estimation of $\|B(t)\|_{c^{1}}$ is omitted since it is highly similar and clearly leads to the same result. Thus if we let

then

$$
Q(t)=\|E(t)\|_{c^{1}}+\|B(t)\|_{c^{1}},
$$

$$
Q(t) \leqq C(t)\left(\varepsilon+\int_{0}^{t} Q(s) d s\right) .
$$

In order to apply Gronwall's inequality we fix $T \in\left[0, T_{\varepsilon}\right)$. Then for all $t \in[0, T]$,

Hence (by Gronwall)

$$
Q(t) \leqq C(T)\left[\varepsilon+\int_{0}^{t} Q(s) d s\right]
$$

$$
Q(t) \leqq C(T) \varepsilon \exp (C(T) t)
$$

for all $t \in[0, T]$. In particular

$$
Q(T) \leqq C(T) \varepsilon \exp (C(T) T)=C(T) \varepsilon,
$$

and the proof is complete.

\section{$\mathrm{C}^{0}$-Estimates Without Neutrality}

The estimates of this section rely on dispersion and will be used for large values of $t$ only. $E$ and $B$ are estimated first; their spatial derivatives will be estimated in the next section.

To estimate $E_{T}$ note first that by Lemma 3,

$$
\begin{aligned}
& \left|\int(1+\hat{u} \cdot w)^{-2}\left(1-|\hat{u}|^{2}\right)(\hat{u}+w)\left(\sum_{\alpha} e_{\alpha} m_{\alpha}^{3} g_{\alpha}(t-|x-y|, y, u)\right) d u\right| \\
& \quad \leqq C \int_{\mathscr{S}(t-|x-y|, y)} \sum_{\alpha}\left|f_{\alpha}(t-|x-y|, y, v)\right| d v \leqq C(1+t-|x-y|)^{-3} \mathscr{X}(t-|x-y|, y)
\end{aligned}
$$


for $0 \leqq|x-y| \leqq t<T_{\varepsilon}$. Note that any possible cancellation in the $\alpha$ summation has already been discarded. Now by (8)

$$
\left|E_{T}\right| \leqq C \int_{|x-y|<t}|x-y|^{-2}(1+t-|x-y|)^{-3} \mathscr{X}(t-|x-y|, y) d y .
$$

On the support of $\mathscr{X}$, where $|y|<t-|x-y|+k$,

$$
1+k+t-|x-y|+|y| \leqq C(1+t-|x-y|)
$$

so

$$
\left|E_{T}\right| \leqq C \int_{|x-y|<t}|x-y|^{-2}(1+k+t-|x-y|+|y|)^{-3} d y
$$

for $0 \leqq t<T_{\varepsilon}$.

To simplify (44) we will use the following Lemma, which is Lemma 7 of [5]. The proof appears in [5] and is not included here.

Lemma 8. For any continuous functions $g(\tau, \lambda)$ of two real variables, and $h(\sigma)$ of one real variable,

$$
\int_{|x-y|<t} g(t-|x-y|,|y|) h(|x-y|) d y=\frac{2 \pi}{|x|} \int_{0}^{t} \int_{|| x|-t+\tau|}^{|x|+t-\tau} g(\tau, \lambda) \lambda d \lambda(t-\tau) h(t-\tau) d \tau,
$$

where the integration on the left is over a ball in $\mathbb{R}^{3}$.

Lemma 9. For all $x \in \mathbb{R}^{3}$ and $t \geqq 0$ with $|x| \leqq t+k$,

$$
\int_{|x-y|<t}|x-y|^{-2}(1+k+t-|x-y|+|y|)^{-3} d y \leqq C(1+k+t+r)^{-1}(1+k+t-r)^{-1} .
$$

Proof. By Lemma 8

$$
\begin{aligned}
& \int_{|x-y|<t}|x-y|^{-2}(1+k+t-|x-y|+|y|)^{-3} d y \\
& =\frac{2 \pi}{|x|} \int_{0}^{t} \int_{|| x|-t+\tau|}^{|x|+t-\tau}(1+k+\tau+\lambda)^{-3} \lambda d \lambda(t-\tau)^{-1} d \tau .
\end{aligned}
$$

We denote

and

$$
r=|x|
$$

$$
(t-r)_{+}=\left\{\begin{array}{cll}
t-r & \text { if } & t-r \geqq 0 \\
0 & \text { if } & t-r<0 .
\end{array}\right.
$$

Case 1. Assume $r<\frac{1}{2}(1+k+t)$. If $0<\tau<t-r$, then

$$
\lambda(t-\tau)^{-1} \leqq(t-\tau+r)(t-\tau)^{-1} \leqq(t-\tau+t-\tau)(t-\tau)^{-1}=2 .
$$

Hence

$$
\begin{aligned}
& \int_{0}^{t} \int_{|r-t+\tau|}^{r+t-\tau}(1+k+\tau+\lambda)^{-3} \lambda d \lambda(t-\tau)^{-1} d \tau \\
& \leqq 2 \int_{0}^{(t-r)^{+}} \int_{t-\tau-r}^{t-\tau+r}(1+k+\tau+\lambda)^{-3} d \lambda d \tau \\
& \quad+\int_{(t-r)_{+}}^{t} \int_{r-t+\tau}^{r+t-\tau}(1+k+\tau+\lambda)^{-2} d \lambda(t-\tau)^{-1} d \tau
\end{aligned}
$$




$$
\begin{aligned}
& \leqq 2 \int_{0}^{(t-r)_{+}} 2 r(1+k+\tau+[t-\tau-r])^{-3} d \tau+2 \int_{(t-r)_{+}}^{t}(1+k+\tau+[r-t+\tau])^{-2} d \tau \\
& \leqq 4 r(t-r)_{+}(1+k+t-r)^{-3}+2 r(1+k+t-r)^{-2} \\
& \leqq 6 r(1+k+t-r)^{-2} \leqq 6 r\left(1+k+t-\frac{1}{2}[1+k+t]\right)^{-2} \\
& \leqq C r(1+k+t+r)^{-1}(1+k+t-r)^{-1}
\end{aligned}
$$

Case 2. Assume $r \geqq \frac{1}{2}(1+k+t)$, then

$$
\begin{aligned}
& \int_{0}^{t} \int_{|r-t+\tau|}^{r+t-\tau}(1+k+\tau+\lambda)^{-3} \lambda d \lambda(t-\tau)^{-1} d \tau \\
& \quad \leqq \int_{0}^{t} \int_{|r-t+\tau|}^{r+t-\tau}(1+k+\tau+\lambda)^{-2} d \lambda(t-\tau)^{-1} d \tau \\
& \quad \leqq 2 \int_{0}^{t}(1+k+\tau+|r-t+\tau|)^{-2} d \tau=2 \int_{0}^{(t-r)_{+}}(1+k+t-r)^{-2} d \tau \\
& \quad+2 \int_{(t-r)_{+}}^{t}(1+k+2 \tau+r-t)^{-2} d \tau \\
& \quad \leqq 2(1+k+t-r)^{-2}(t-r)_{+}+\left(1+k+2[t-r]_{+}+r-t\right)^{-1} \\
& \quad \leqq C(1+k+t-r)^{-1} \leqq C r(1+k+t+r)^{-1}(1+k+t-r)^{-1} .
\end{aligned}
$$

Now, if we use (46) and (47) in (45) we obtain the desired result.

Lemma 10. For $0 \leqq t<T_{\varepsilon}$,

$$
\left|E_{T}\right|+\left|B_{T}\right| \leqq C(1+k+t+|x|)^{-1}(1+k+t-|x|)^{-1} \mathscr{X}(t, x) .
$$

Proof. Note first that $E_{T} \equiv 0$ on $|x|>k+t$ by (8) and the support property of $g_{\alpha}$. The estimate for $E_{T}$ now follows from Lemma 9 and (44). The estimate for $B_{T}$ is similar.

To estimate $E_{S}$ and $B_{S}$ we will use the following lemma which is a space-time version of Gronwall's inequality.

Lemma 11. Given $T_{0}>0$, define

$$
\mathscr{C}=C^{0}\left(\left\{(t, x): 0 \leqq t \leqq T_{0}, x \in \mathbb{R}^{3},|x| \leqq k+t\right\}\right) .
$$

Given $D>0, A>0, p \in(0,1]$, and $q \in[0, p+1]$, define $\mathscr{L}: \mathscr{C} \rightarrow \mathscr{C}$ by

$$
\begin{aligned}
\mathscr{L}(u)= & A(1+k+t+|x|)^{-p}(1+k+t-|x|)^{-q} \\
& +\int_{|x-y|<t} \mathscr{X}(t-|x-y|, y)|x-y|^{-1} D(1+k+t-|x-y|+|y|)^{-3} \\
& \cdot u(t-|x-y|, y) d y .
\end{aligned}
$$

If $v \in \mathscr{C}$ satisfies $v \leqq \mathscr{L}(v)($ on $|x| \leqq k+t)$, then

$$
v \leqq 2 A e^{C D} p^{-1}(1+k+t+|x|)^{-p}(1+k+t-|x|)^{-q}
$$

on $|x| \leqq k+t$.

Proof. Standard techniques show that $\mathscr{L}$ has a unique fixed point $u_{f} \in \mathscr{C}$, and that 
$\mathscr{L}^{n}(u) \rightarrow u_{f}$ for every $u \in \mathscr{C}$. We will construct $z \in \mathscr{C}$ such that $\mathscr{L}(z) \leqq z$. Then

$$
v \leqq \mathscr{L}(v) \leqq \mathscr{L}^{2}(v) \leqq \cdots \leqq \mathscr{L}^{n}(v) \nearrow u_{f}
$$

and

$$
u_{f} \mathscr{L}^{n}(z) \leqq \cdots \leqq \mathscr{L}^{2}(z) \leqq \mathscr{L}(z) \leqq z .
$$

In particular $v \leqq z$, which will give the desired inequality.

Take $r=|x| \leqq t+k$ and

where

$$
z(t, x)=\alpha z_{1}(t+r) z_{2}(t-r)
$$

$$
\begin{aligned}
& z_{1}(\xi)=(1+k+\xi)^{-p} \\
& z_{2}(\eta)=(1+k+\eta)^{-q} \exp \left[\beta D\left(1-[1+k+\eta]^{-p}\right)\right]
\end{aligned}
$$

and $\alpha, \beta$ are to be chosen later. By Lemma 8

$$
\begin{aligned}
& \int_{|x-y|<t} \mathscr{X}(t-|x-y|, y)|x-y|^{-1} D(1+k+t-|x-y|+|y|)^{-3} z(t-|x-y|, y) d y \\
& =\frac{2 \pi}{r} \int_{0}^{t} \int_{|r-t+\tau|}^{r+t-\tau} \mathscr{X}(\tau, \lambda)(t-\tau)^{-1} D(1+k+\tau+\lambda)^{-3} \alpha z_{1}(\tau+\lambda) z_{2}(\tau-\lambda) \lambda d \lambda(t-\tau) d \tau,
\end{aligned}
$$

where we are abusing notation by writing

$$
\mathscr{X}(\tau, y)=\mathscr{X}(\tau,|y|) .
$$

Making the change of variables

$$
\xi=\tau+\lambda, \quad \eta=\tau-\lambda,
$$

we obtain

$$
\begin{array}{rl}
\int_{|x-y|<t} & X(t-|x-y|, y)|x-y|^{-1} D(1+k+t-|x-y|+|y|)^{-3} z(t-|x-y|, y) d y \\
& \leqq C D \alpha r^{-1} \int_{t-r}^{t+r}(1+k+\xi)^{-3} z_{1}(\xi) \int_{-k}^{t-r} z_{2}(\eta)(\xi-\eta) d \eta d \xi \\
& \leqq C D \alpha r^{-1}\left(\int_{t-r}^{t+r}(1+k+\xi)^{-2-p} d \xi\right)\left(\int_{-k}^{t-r} z_{2}(\eta) d \eta\right) .
\end{array}
$$

To estimate the $\xi$ integral note that if $r>\frac{1}{2}(1+k+t)$ then

$$
\begin{aligned}
r^{-1} \int_{t-r}^{t+r}(1+k+\xi)^{-2-p} d \xi & \leqq \frac{2}{1+k+t} \frac{1}{1+p}(1+k+t-r)^{-1-p} \\
& \leqq C(1+k+t+r)^{-1}(1+k+t-r)^{-1-p}
\end{aligned}
$$

If $r \leqq \frac{1}{2}(1+k+t)$

$$
\begin{aligned}
& r^{-1} \int_{t-r}^{t+r}(1+k+\xi)^{-2-p} d \xi \leqq r^{-1}(2 r)(1+k+t-r)^{-2-p} \\
& \quad \leqq 2\left(1+k+t-\frac{1}{2}[1+k+t]\right)^{-1}(1+k+t-r)^{-1-p} \\
& \quad \leqq C(1+k+t+r)^{-1}(1+k+t-r)^{-1-p}
\end{aligned}
$$


For the $\eta$ integral in (48) note that

$$
\begin{aligned}
& \int_{-k}^{t-r} z_{2}(\eta) d \eta \\
& \quad \leqq(1+k+t-r)^{p+1-q} \int_{-k}^{t-r}(1+k+\eta)^{-p-1} \exp \left[\beta D\left(1-[1+k+\eta]^{-p}\right)\right] d \eta \\
& \quad=\left.(1+k+t-r)^{p+1-q}(\beta D p)^{-1} \exp \left[\beta D\left(1-[1+k+\eta]^{-p}\right)\right]\right|_{-k} ^{t-r} \\
& \quad \leqq(1+k+t-r)^{p+1-q}(\beta D p)^{-1} \exp \left[\beta D\left(1-[1+k+t-r]^{-p}\right)\right] .
\end{aligned}
$$

Now we substitute (49), (50), and (51) into (48) to obtain

$$
\begin{array}{rl}
\int_{|x-y|<t} & X(t-|x-y|, y)|x-y|^{-1} D(1+k+t-|x-y|+|y|)^{-3} z(t-|x-y|, y) d y \\
\leqq & C D \alpha\left[C(1+k+t+r)^{-1}(1+k+t-r)^{-1-p}\right] \\
& \cdot(1+k+t-r)^{p+1-q}(\beta D p)^{-1} \exp \left[\beta D\left(1-[1+k+t-r]^{-p}\right)\right] \\
= & C \beta^{-1} p^{-1}(1+k+t+r)^{p-1} z .
\end{array}
$$

Hence

$$
\mathscr{L}(z) \leqq A(1+k+t+r)^{-p}(1+k+t-r)^{-q}+C \beta^{-1} p^{-1} z .
$$

Now we take $\alpha=2 \mathrm{~A}$ so that

$$
A(1+k+t+r)^{-p}(1+k+t-r)^{-q} \leqq \frac{1}{2} z .
$$

and hence by (52)

$$
\mathscr{L}(z) \leqq\left(\frac{1}{2}+C \beta^{-1} p^{-1}\right) z .
$$

Finally taking $\beta=2 C p^{-1}$, we get

$$
\mathscr{L}(z) \leqq z,
$$

so (as shown before)

$$
\begin{aligned}
v & \leqq z=2 \mathrm{~A}(1+k+t+r)^{-p}(1+k+t-r)^{-q} \exp \left[\frac{2 C D}{p}\left(1-[1+k+t-r]^{-p}\right)\right] \\
& \leqq 2 \mathrm{~A} \exp \left(C D p^{-1}\right)(1+k+t+r)^{-p}(1+k+t-r)^{-q} .
\end{aligned}
$$

The proof is now complete.

Lemma 12. There exists a function $D:\left(0, \eta_{2}\right) \rightarrow(0, \infty)$ such that

and

$$
\lim _{\varepsilon \rightarrow 0^{+}} D(\varepsilon)=0
$$

$$
|E(t, x)|+|B(t, x)| \leqq D(\varepsilon)(1+k+t+|x|)^{-3 / 4}(1+k+t-|x|)^{-3 / 4} \mathscr{X}(t, x)
$$

for $0 \leqq t<T_{\varepsilon}$.

Proof. Note first that by Lemmas 4, 7, and 10,

$$
\begin{aligned}
\left|E_{z}\right| & +\left|B_{z}\right|+\left|E_{T}\right|+\left|B_{T}\right| \leqq C \varepsilon(1+k+t+|x|)^{-1}(1+k+t-|x|)^{-1} \mathscr{X}(t, x) \\
& +\min \left\{C(t) \varepsilon, C(1+k+t+|x|)^{-1}(1+k+t-|x|)^{-1} \mathscr{X}(t, x)\right\}
\end{aligned}
$$


for $0 \leqq t<T_{\varepsilon}$. Before proceeding let us simplify this estimate. Writing $r=|x|$ and taking $r \leqq k+t$, we note that

$$
\begin{aligned}
& (1+k+t+r)^{3 / 4}(1+k+t-r)^{3 / 4} \min \left\{C(t) \varepsilon, \quad C(1+k+t+r)^{-1}(1+k+t-r)^{-1}\right\} \\
& \quad \leqq \min \left\{C(t) \varepsilon(1+2 k+2 t)^{3 / 2}, \quad C(1+k+t+r)^{-1 / 4}(1+k+t-r)^{-1 / 4}\right\} \\
& \quad \leqq \min \left\{C(t) \varepsilon, \quad C(1+k+t)^{-1 / 4}\right\} \leqq C \min \left\{C(t) \varepsilon, t^{-1 / 4}\right\} .
\end{aligned}
$$

Define $D(\varepsilon)$ to be the maximal value of the mapping

$$
t \in(0, \infty) \mapsto C \min \left\{C(t) \varepsilon, t^{-1 / 4}\right\} .
$$

Note that

$$
\lim _{\varepsilon \rightarrow 0^{+}} D(\varepsilon)=0
$$

and that by (54)

$$
\begin{gathered}
\min \left\{C(t) \varepsilon, \quad C(1+k+t+r)^{-1}(1+k+t-r)^{-1}\right\} \\
\leqq D(\varepsilon)(1+k+t+r)^{-3 / 4}(1+k+t-r)^{-3 / 4}
\end{gathered}
$$

for $r \leqq k+t$. Hence by (53),

$$
\left|E_{z}\right|+\left|B_{z}\right|+\left|E_{T}\right|+\left|B_{T}\right| \leqq(C \varepsilon+D(\varepsilon))(1+k+t+|x|)^{-3 / 4}(1+k+t-|x|)^{-3 / 4} \mathscr{X}(t, x)
$$

for $0 \leqq t<T_{\varepsilon}$.

Now we consider $E_{S}$. By (9)

$$
\left|E_{S}\right| \leqq\left.\int_{|x-y|<t}|x-y|^{-1} \int\left|\nabla_{u}\left([1+\hat{u} \cdot w]^{-1}[w+\hat{u}]\right)\right|\left|\left(\sum_{\alpha} e_{\alpha}^{2} m_{\alpha}^{2} g_{\alpha}\right) K\right|\right|_{(t-|x-y|, y, u)} d u d y
$$

By Lemma 1 the $u$ support is bounded for $0 \leqq t<T_{\varepsilon}$, so by Lemma 3

$$
\begin{aligned}
\left|E_{S}\right| \leqq & C \int_{|x-y|<t}|x-y|^{-1}\left(\int_{\mathscr{S}(t-|x-y|, y)} \sum_{\alpha} f_{\alpha}(t-|x-y|, y, v) d v\right) \\
& \left.\cdot(|E|+|B|)\right|_{(t-|x-y|, y)} d y \\
\leqq & C \int_{|x-y|<t}|x-y|^{-1} \mathscr{X}(t-|x-y|, y) C(1+t-|x-y|)^{-3} \\
& \left.\cdot(|E|+|B|)\right|_{(t-|x-y|, y)} d y .
\end{aligned}
$$

Note that for $|y| \leqq t-|x-y|+k$,

$$
(1+t-|x-y|)^{-3} \leqq C(1+k+t-|x-y|+|y|)^{-3} .
$$

The same analysis of $B_{S}$ reveals that $\left|B_{S}\right|$ is bounded by the right-hand side of (57). hence (for $0 \leqq t<T_{\varepsilon}$ ),

$$
\begin{aligned}
\left|E_{S}\right|+\left|B_{S}\right| \leqq & C \int_{|x-y|<t}|x-y|^{-1} \mathscr{X}(t-|x-y|, y) \\
& \left.\cdot(1+k+t-|x-y|+|y|)^{-3}(|E|+|B|)\right|_{(t-|x-y|, y)} d y .
\end{aligned}
$$


Now using (56) and (58) in the representations (6) and (6B) we get

$$
\begin{aligned}
|E|+|B| \leqq & (C \varepsilon+D(\varepsilon))(1+k+t+|x|)^{-3 / 4}(1+k+t-|x|)^{-3 / 4} \mathscr{X}(t, x) \\
& +C \int_{|x-y|<t}|x-y|^{-1} \mathscr{X}(t-|x-y|, y)(1+k+t-|x-y|+|y|)^{-3} \\
& \left.\cdot(|E|+|B|)\right|_{(t-|x-y|, y)} d y .
\end{aligned}
$$

We now apply Lemma 11 , with $q=p=3 / 4$, and obtain

$$
|E(t, x)|+|B(t, x)| \leqq 2(C \varepsilon+D(\varepsilon)) e^{C}(1+k+t+|x|)^{-3 / 4}(1+k+t-|x|)^{-3 / 4}
$$

for $0 \leqq t<T_{\varepsilon}$ and $|x| \leqq t+k$. Now since $E$ and $B$ vanish on $|x|>t+k$, the lemma follows.

Corollary to Lemma 12. For $0 \leqq t<T_{\varepsilon}$, we have

$$
\|j(t)\|_{c^{0}} \leqq C D(\varepsilon)(1+t)^{-7 / 4},
$$

where $D(\varepsilon)$ is as in Lemma 12.

Proof. By Lemmas 6 and 12 we have

$$
\begin{aligned}
\left\|\sum_{\alpha} e_{\alpha} m_{\alpha}^{3} g_{\alpha}(t)\right\|_{c^{0}} & \leqq \varepsilon+C \int_{0}^{t}(1+s)\left[\|E(s)\|_{c^{0}}+\|B(s)\|_{c^{0}}\right] d s \\
& \leqq \varepsilon+C D(\varepsilon) \int_{0}^{t}(1+s)^{1 / 4} d s \leqq C D(\varepsilon)(1+t)^{5 / 4}
\end{aligned}
$$

Now using Lemma 3 we have

$$
|j(t, x)| \leqq C(1+t)^{-3}\left\|\sum_{\alpha} e_{\alpha} m_{\alpha}^{3} g_{\alpha}(t)\right\|_{c^{0}} \leqq C D(\varepsilon)(1+t)^{-7 / 4} .
$$

\section{$C^{1}$-Estimates Without Neutrality}

Our goal in this section is to obtain the analogue of Lemma 12 for derivatives of the fields. Explicitly, we will show that there exists a function $\tilde{D}(\varepsilon)$ mapping $0<\varepsilon<\eta_{2}$ into $(0, \infty)$ with the properties that $\widetilde{D}(\varepsilon)=o(1)$ as $\varepsilon \rightarrow 0$ and on $0 \leqq t<T_{\varepsilon}$,

$$
\left|E_{x}(t, x)\right|+\left|B_{x}(t, x)\right| \leqq \tilde{D}(\varepsilon)(1+k+t+|x|)^{-3 / 4}(1+k+t-|x|)^{-7 / 4} \mathscr{X}(t, x) .
$$

This will enable us to establish the validity of the (FSC) for all times $t>0$.

We begin with the representation (10):

$$
\frac{\partial}{\partial x_{k}} E^{i}=A_{z}+A_{w}+A_{T T}+A_{T S}+A_{S T}+A_{S S}
$$

where the various terms are given in (11)-(13), (15)-(16). We will estimate $\partial E^{i} / \partial x_{k}$ only, since the estimates for $\partial B^{i} / \partial x_{k}$ are nearly identical.

By Lemma 4, we already know that

$$
\left|A_{z}\right| \leqq C \varepsilon(1+k+t+|x|)^{-1}(1+k+t-|x|)^{-2} \mathscr{X}(t, x) .
$$

In order to estimate $A_{T T}$, we require 
Lemma 13. Let $\eta_{2}$ be chosen as in Lemma 2, and let the (FSC) hold for $0<\eta \leqq \eta_{2}$, $0<t<T_{\varepsilon}$. Then there exists a constant $C$ depending on $k, \eta_{2}, e_{\alpha}, m_{\alpha}$ (but independent of $t$ ) such that for all $u \in \mathbb{R}^{3},|x| \leqq k+t, 0 \leqq t<T_{\varepsilon}$ we have

$$
\begin{aligned}
& \max _{\alpha}\left|\nabla_{x} g_{\alpha}(t, x, u)\right| \leqq C \max _{\alpha}\left\|g_{\alpha}^{0}\right\|_{c^{1}}, \\
& \max _{\alpha}\left|\nabla_{u} g_{\alpha}(t, x, u)\right| \leqq C(1+t) \max _{\alpha}\left\|g_{\alpha}^{0}\right\|_{c^{1}} .
\end{aligned}
$$

Proof. This is a slightly modified version of Lemma 5 of [5]. Indeed, the (FSC) shows that the kernel $I(s)$ (appearing in (25) of [5]) now becomes $(1+s)^{-5 / 2}$. The pointwise inequality for $\nabla_{u} g_{\alpha}$ appearing above Eq. (26) of [5] now has the kernel $c(1+s)^{-3 / 2}$, which is integrable. The argument is concluded by simply noting that $(1+s) I(s)=c(1+s)^{-3 / 2}$ remains integrable.

Now we can estimate $A_{w}$ and $A_{T T}$ :

Lemma 14. Let $r=|x|$. There exists a constant $C$ independent of $t$ such that for $r \leqq t+k, 0 \leqq t<T_{\varepsilon}$,

$$
\left|A_{w}\right|+\left|A_{T T}\right| \leqq C(1+k+t+r)^{-1}(1+k+t-r)^{-2} \log (1+k+t+r) \mathscr{X}(t, r) .
$$

Proof. From (11) we have

$$
A_{T T}=\int_{|x-y|<t}|y-x|^{-3} \int a(w, \hat{u})\left(\sum_{\alpha} e_{\alpha} m_{\alpha}^{3} g_{\alpha}(t-|x-y|, y, u)\right) d u d y,
$$

and the kernel $a$ has zero mean value over $|w|=1$ by (14). Consider the integral $A_{T T}^{(1)}$ defined to be that taken over the set $1 \leqq|y-x| \leqq t$. We have

$$
\begin{aligned}
\left|A_{T T}^{(1)}\right| & \leqq C \int_{1 \leqq|y-x| \leqq t} \mathscr{X} \cdot|y-x|^{-3} \int_{\mathscr{S}(t-|x-y|, y)} \sum_{\alpha}\left|f_{\alpha}(t-|x-y|, y, v)\right| d v \\
& \leqq C \int_{1 \leqq|x-y| \leqq t} \frac{\mathscr{X}(t-|x-y|, y) d y}{|y-x|^{3}(1+t-|x-y|)^{3}},
\end{aligned}
$$

where we have used Lemma 3 to bound the measure of $\mathscr{S}$. On the support of $\mathscr{X}$, where

$$
|y| \leqq k+t-|x-y|
$$

we have

$$
1+k+t-|x-y|+|y| \leqq C(1+t-|x-y|)
$$

hence

$$
\left|A_{T T}^{(1)}\right| \leqq C \int_{1 \leqq|y-x| \leqq t} \frac{\mathscr{X}(t-|x-y|, y) d y}{|y-x|^{3}(1+k+t-|x-y|+|y|)^{3}},
$$

and by applying Lemma 8 we arrive at

$$
\left|A_{T T}^{(1)}\right| \leqq C r^{-1} \int_{0}^{t-1} \frac{d \tau}{(t-\tau)^{2}} \int_{|r-(t-\tau)|}^{r+t-\tau} \frac{\mathscr{X}(\tau, \lambda) \lambda d \lambda}{(1+k+\tau+\lambda)^{3}} .
$$

This integral is split up into integrals over $[0, t / 2]$ and $[t / 2, t-1]$. We assume 
without loss of generality that $t$ is large. Then

$$
\begin{aligned}
\int_{0}^{t / 2} & \leqq \frac{4}{t^{2}} \int_{0}^{t / 2} d \tau \int_{|r-t+\tau|}^{r+t-\tau} \frac{d \lambda}{(1+k+\tau+\lambda)^{2}} \\
& =\frac{4}{t^{2}} \int_{0}^{t / 2} \frac{(t+r-\tau)-|r-t+\tau|}{(1+k+t+r)(1+k+\tau+|r-t+\tau|)} d \tau \\
& \leqq \frac{4}{t^{2}(1+k+t+r)}\left\{\int_{0}^{(t-r)_{+}} \frac{2 r}{1+k+t-r} d \tau+\int_{(t-r)_{+}}^{t} \frac{2(t-\tau) d \tau}{1+k+2 \tau-t+r}\right\} \\
& \leqq \frac{4}{t^{2}(1+k+t+r)}\left\{2 r+\frac{1}{1+k+t-r} \int_{(t-r)_{+}}^{t} 2(t-\tau) d \tau\right\} \\
& \leqq C r(1+k+t+r)^{-2}(1+k+t-r)^{-1},
\end{aligned}
$$

since $r \leqq t+k$. For the integral over the complement we have

$$
\begin{aligned}
\int_{t / 2}^{t-1} & \leqq\left(1+k+\frac{t}{2}\right)^{-3} \int_{t / 2}^{t-1} \int_{|r-t+\tau|}^{r+t-\tau} \lambda d \lambda \frac{d \tau}{(t-\tau)^{2}}=2 r\left(1+k+\frac{t}{2}\right)^{-3 t-1} \int_{t / 2}^{t} \frac{d \tau}{t-\tau} \\
& \leqq C r(1+k+t)^{-3} \cdot \log \left(\frac{t}{2}\right) \leqq C r(1+k+t+r)^{-3} \log (1+k+t+r),
\end{aligned}
$$

since again $r \leqq t+k$. Using (62) and (63) in (61) we certainly get

$$
\left|A_{T T}^{(1)}\right| \leqq C(1+k+t+r)^{-1}(1+k+t-r)^{-2} \log (1+k+t+r) \cdot \mathscr{X}(t, r) .
$$

Returning to the formula (11) for $A_{T T}$, we can write the integral over the set $|y-x|<1$ as

$$
\begin{aligned}
\left|A_{T T}^{(2)}\right| \equiv & \left|\int_{|x-y|<1}\right| y-\left.x\right|^{-3} \int a(w, \hat{u}) \sum_{\alpha} e_{\alpha} m_{\alpha}^{3}\left[g_{\alpha}(t-|x-y|, y, u)\right. \\
& \left.-g_{\alpha}(t-|x-y|, x, u)\right] d u d y \mid
\end{aligned}
$$

since $\int_{|w|=1} a d w=0$ by (14). Again, by Lemma 3, the $u$-integration is taken only over a set of measure less than $C(2+t-|x-y|)^{-3}$. Now we apply the mean-value theorem to the integrand of $A_{T T}^{(2)}$, noting that $\left|\nabla_{x} g_{\alpha}(t, x, u)\right|$ is uniformly bounded by $C\left\|g_{\alpha}^{0}\right\|_{c^{1}}$ in view of Lemma 13 . Thus

$$
\left|A_{T T}^{(2)}\right| \leqq C \max _{\alpha}\left\|g_{\alpha}^{0}\right\|_{c^{1}} \int_{|x-y|<1} \frac{\mathscr{X}(t-|x-y|, y) d y}{|y-x|^{2}(2+t-|x-y|)^{3}} .
$$

For large $t$, this integral is clearly dominated by $C t^{-3}$, and hence also by $C(1+k+t+r)^{-3}$, hence also by

$$
C(1+k+t+r)^{-1}(1+k+t-r)^{-2} \cdot \mathscr{X}(t, r)
$$

since $r \leqq t+k$. Using this bound and (64), we see that

$$
\left|A_{T T}\right| \leqq C(1+k+t+r)^{-1}(1+k+t-r)^{-2} \log (1+k+t+r) \cdot \mathscr{X} .
$$


This is the desired estimate for $A_{T T}$.

Turning to $A_{w}$, we have from (16)

$$
A_{w}=-\int d(w, \hat{u}) \sum_{\alpha} e_{\alpha} m_{\alpha}^{3} g_{\alpha}(t, x, u) d u .
$$

By Lemma 1, the kernel $d$ is uniformly bounded, as are the $g_{\alpha}$ 's. By Lemma 3, the $u$-integration in (16) is taken only over a set of measure less than $C(1+t)^{-3}$. Therefore $A_{w}$ satisfies the same estimate as $A_{T T}$, so we have proven Lemma 14.

Corollary to Lemma 14. For $r \leqq t+k$ and $0 \leqq t<T_{\varepsilon}$ we have

$$
\left|A_{z}\right|+\left|A_{w}\right|+\left|A_{T T}\right| \leqslant C(1+k+t+r)^{-1}(1+k+t-r)^{-2} \log (1+k+t+r) .
$$

This follows immediately from (60).

Lemma 15. There exists a function $D_{1}(\varepsilon)$ on $\left(0, \eta_{2}\right)$ into $(0, \infty)$ such that

$$
\begin{gathered}
D_{1}(\varepsilon)=o(1) \quad \text { as } \quad \varepsilon \rightarrow 0 \text {, and } \\
\left|A_{z}\right|+\left|A_{w}\right|+\left|A_{T T}\right| \leqq D_{1}(\varepsilon)(1+k+t+r)^{-3 / 4}(1+k+t-r)^{-7 / 4} \mathscr{X}(t, r)
\end{gathered}
$$

for $0 \leqq t<T_{\varepsilon}$.

Proof. By Lemma 4, 7, and 14, we have

$$
\begin{aligned}
\left|A_{z}\right|+\left|A_{w}\right|+\left|A_{T T}\right| \leqq & C \min \left\{C(t) \varepsilon,(1+k+t+r)^{-1}\right. \\
& \left.\cdot(1+k+t-r)^{-2} \log (1+k+t+r)\right\}
\end{aligned}
$$

for $r \leqq k+t$. Note that for $r \leqq k+t$,

$$
\begin{aligned}
& (1+k+t+r)^{3 / 4}(1+k+t-r)^{7 / 4} \min \{C(t) \varepsilon, \\
& \left.\quad \cdot(1+k+t+r)^{-1}(1+k+t-r)^{-2} \log (1+k+t+r)\right\} \\
& \quad \leqq C \min \left\{\widetilde{C}(t) \varepsilon, t^{-1 / 8}\right\} .
\end{aligned}
$$

Define $D_{1}(\varepsilon)$ to be the maximal value of the mapping

Note that

$$
t \mapsto \min \left\{\tilde{C}(t) \varepsilon, t^{-1 / 8}\right\} \text {. }
$$

$$
\lim _{\varepsilon \rightarrow 0+} D_{1}(\varepsilon)=0
$$

and also

$$
\begin{aligned}
& \min \left\{C(t) \varepsilon,(1+k+t+r)^{-1}(1+k+t-r)^{-2} \log (1+k+t+r)\right\} \\
& \quad \leqq C D_{1}(\varepsilon)(1+k+t+r)^{-3 / 4}(1+k+t-r)^{-7 / 4}
\end{aligned}
$$

for $r \leqq t+k$. Hence

$$
\left|A_{z}\right|+\left|A_{w}\right|+\left|A_{T T}\right| \leqq C D_{1}(\varepsilon)(1+k+t+r)^{-3 / 4}(1+k+t-r)^{-7 / 4}
$$

for $r \leqq t+k$. But

$$
\left|A_{z}\right|+\left|A_{w}\right|+\left|A_{T T}\right|=0
$$

if $r>k+t$, so the proof is complete. 
Lemma 16. There exists a function $D_{2}(\varepsilon)$ on $\left(0, \eta_{2}\right)$ into $(0, \infty)$ such that

$$
\begin{gathered}
D_{2}(\varepsilon)=o(1) \quad \text { as } \quad \varepsilon \rightarrow 0, \text { and } \\
\left|A_{S T}+A_{T S}\right| \leqq D_{2}(\varepsilon)(1+k+t+r)^{-3 / 4}(1+k+t-r)^{-7 / 4} \mathscr{X}(t, r)
\end{gathered}
$$

for $0<t<T_{\varepsilon}$.

Proof. By Eq. (12) we can write

$$
A_{T S}+A_{S T}=\int_{|x-y|<t}|x-y|^{-2} \int b(w, \hat{u})\left(\sum_{\alpha} e_{\alpha} m_{\alpha}^{3} S g_{\alpha}(t-|x-y|, y, u)\right) d u d y,
$$

where the kernel $b$ is bounded for bounded $u$, as is the case by Lemma 1 . Since $S g_{\alpha}$ is the $u$-divergence of $-e_{\alpha} m_{\alpha}^{-1} K g_{\alpha}$, we can integrate (12) by parts in $u$ (as was done in (36)) to obtain, with $\mathscr{X}=\mathscr{X}(t-|x-y|, y)$,

$$
\begin{aligned}
\left|A_{T S}+A_{S T}\right| \leqq & C \int_{|x-y|<t}|x-y|^{-2} \mathscr{X} \\
& \cdot \int_{\mathscr{S}(t-|x-y|, y)} \sum_{\alpha}|K(t-|x-y|, y)| f_{\alpha}(t-|x-y|, y, v) d v d y .
\end{aligned}
$$

By Lemma 3, then,

$$
\left|A_{T S}+A_{S T}\right| \leqq C \int_{|x-y|<t}|x-y|^{-2} \mathscr{X}|K(t-|x-y|, y)|(1+t-|x-y|)^{-3} d y .
$$

We may replace $(1+t-|x-y|)^{-3}$ by $C(1+k+t-|x-y|+|y|)^{-3}$ on the support of $\mathscr{X}$, and apply the bound for $K$ from Lemma 12 to get

$$
\begin{aligned}
& \left|A_{T S}+A_{S T}\right| \leqq C \\
& \cdot \int_{|x-y|<t} \frac{\mathscr{X} D(\varepsilon) d y}{|y-x|^{2}(1+k+t-|y-x|+|y|)^{3}(1+k+t-|y-x|+|y|)^{3 / 4}(1+k+t-|x-y|-|y|)^{3 / 4}},
\end{aligned}
$$

where $D(\varepsilon)=o(1)$ as $\varepsilon \rightarrow 0$. The factor $1+k+t-|x-y|-|y|$ exceeds unity on the support of $\mathscr{X}$, and moreover

$$
1+k+t-|x-y|+|y| \geqq 1+k+t-r,
$$

so that

$$
\begin{aligned}
\left|A_{T S}+A_{S T}\right| & \leqq C D(\varepsilon)(1+k+t-r)^{-3 / 4} \int_{|x-y|<t} \frac{\mathscr{X}(t-|x-y|, y) d y}{|y-x|^{2}(1+k+t-|y-x|+|y|)^{3}} \\
& \leqq C D(\varepsilon)(1+k+t+r)^{-1}(1+k+t-r)^{-7 / 4} \mathscr{X}(t, r),
\end{aligned}
$$

where we have used Lemma 9 in the last line. This is stronger than what is required in Lemma 16.

Lemma 17. For all $x \in \mathbb{R}^{3}, t \geqq 0, p \geqq 3$, and $q \in[0,1)$ we have

$$
\begin{aligned}
\mathscr{J} \equiv & \int_{|x-y|<t}|x-y|^{-1}(1+k+t-|y-x|+|y|)^{-p} \\
& \cdot(1+k+t-|y-x|-|y|)^{-q} \mathscr{X}(t-|y-x|, y) d y \\
\leqq & C(1-q)^{-1}(1+k+t+r)^{-1}(1+k+t-r)^{3-p-q} \mathscr{X}(t, r) .
\end{aligned}
$$


Proof. By Lemma 8 we have for $|x| \leqq k+t$

$$
\begin{aligned}
\mathscr{J} & =\frac{2 \pi}{r} \int_{0}^{t} \int_{|r-t+\tau|}^{r+t-\tau}(1+k+\tau+\lambda)^{-p}(1+k+\tau-\lambda)^{-q} \mathscr{X}(\tau, \lambda) \lambda d \lambda d \tau \\
& \leqq 2 \pi r^{-1} \int_{0}^{t} \int_{|r-t+\tau|}^{r+t-\tau}(1+k+\tau+\lambda)^{1-p}(1+k+\tau-\lambda)^{-q} \mathscr{X}(\tau, \lambda) d \lambda d \tau .
\end{aligned}
$$

Changing to the variables $\xi=\lambda+\tau$ and $\eta=\tau-\lambda$, we obtain

$$
\begin{aligned}
\mathscr{J} & \leqq C r^{-1} \int_{t}^{t+r} \int_{-k}^{t-r}(1+k+\xi)^{1-p}(1+k+\eta)^{-q} d \eta d \xi \\
& \leqq C r^{-1}(1-q)^{-1}(1+k+t-r)^{1-q} \int_{t-r}^{t+r}(1+k+\xi)^{1-p} d \xi .
\end{aligned}
$$

By the computations in (49) and (50) we see that

$$
r^{-1} \int_{t-r}^{t+r}(1+k+\xi)^{1-p} d \xi \leqq C(1+k+t+r)^{-1}(1+k+t-r)^{2-p},
$$

and hence (for $|x| \leqq k+t)$

$$
\mathscr{J} \leqq C(1-q)^{-1}(1+k+t-r)^{1-q}(1+k+t+r)^{-1}(1+k+t-r)^{2-p} .
$$

The Lemma now follows since $\mathscr{J}=0$ if $|x|>k+t$.

Only the term $A_{S S}$ in the representation (10) for $\left(\partial / \partial x_{k}\right) E^{i}$ has yet to be estimated. From (37), we have

$$
A_{S S}=\mathrm{I}+\mathrm{II}+\mathrm{III}
$$

where the terms are given explicitly beneath (37), and will be written again below. Since the velocities $u$ are bounded by Lemma 1 , the kernels $\left(\partial^{2} / \partial u_{i} \partial u_{j}\right) c(w, \hat{u})$, etc. are uniformly bounded. For the first expression we have

$$
\begin{aligned}
|\mathrm{I}| & =\left|\int_{|y-x|<t}\right| y-\left.x\right|^{-1} \int_{i, j, \alpha} \frac{\partial^{2} c}{\partial u_{i} \partial u_{j}}(w, \hat{u}) e_{\alpha}^{3} m_{\alpha} g_{\alpha} K_{i} K_{j}||_{(t-|x-y|, y, u)} d u d y \mid \\
\leqq & C \int_{|y-x|<t} \frac{d y}{|y-x|} \int_{\mathscr{S}(t-|x-y|,|y|)}|K(t-|x-y|, y)|^{2} \sum_{\alpha} f_{\alpha}(t-|x-y|, y, v) d v \\
\leqq & C \int_{|y-x|<t}|x-y|^{-1} D^{2}(\varepsilon)(1+k+t-|y-x|+|y|)^{-9 / 2} \\
& \cdot(1+k+t-|y-x|-|y|)^{-3 / 2} \mathscr{X}(t-|y-x|, y) d y \\
\leqq & C D^{2}(\varepsilon) \int_{|y-x|<t}|y-x|^{-1}(1+k+t-|y-x|+|y|)^{-9 / 2} \mathscr{X}(t-|y-x|, y) d y,
\end{aligned}
$$

where we have used Lemmas 3 and 12. Now using the fact that $D(\varepsilon)=o(1)$ as $\varepsilon \rightarrow 0$ and Lemma 17 (with $p=9 / 2$ and $q=0$ ) gives us

$$
\begin{aligned}
|\mathrm{I}| & \leqq C D^{2}(\varepsilon)(1+k+t+r)^{-1}(1+k+t-r)^{-3 / 2} \mathscr{X}(t, r) \\
& \leqq C D(\varepsilon)(1+k+t+r)^{-3 / 4}(1+k+t-r)^{-7 / 4} \mathscr{X}(t, r)
\end{aligned}
$$

for $\varepsilon$ sufficiently small. 
Now for terms II and III, we have from (37)

where

$$
\mathrm{II}+\mathrm{III}=\int_{|y-x|<t}|x-y|^{-1} \mathscr{X} \int \sum_{\alpha} e_{\alpha}^{2} m_{\alpha}^{2} H(t-|y-x|, y, u) d u d y,
$$

$$
\begin{aligned}
H(t-|y-x|, y, u)= & \nabla_{u} c(w, \hat{u}) \cdot g_{\alpha}(t-|y-x|, y, u) S K(t-|y-x|, y, u) \\
& +\left.c(w, \hat{u}) \sum_{i, j} \frac{\partial \hat{u}_{j}}{\partial u_{i}} \frac{\partial}{\partial y_{j}}\left(g_{\alpha} K_{i}\right)\right|_{(t-|y-x|, y, u)}
\end{aligned}
$$

By the definition of $S,|S K| \leqq C\left(\left|K_{x}\right|+\left|K_{t}\right|\right)$, and by Maxwell's equations

$$
\left|K_{t}(t, x)\right| \leqq C\left|K_{x}(t, x)\right|+|j(t, x)| .
$$

Moreover, by the Corollary to Lemma 12,

$$
|S K| \leqq C\left(\left|K_{x}(t, x)\right|+D(\varepsilon)(1+t)^{-7 / 4}\right) .
$$

The last term in $H$ involves

$$
\frac{\partial}{\partial y_{j}}\left(g_{\alpha} K_{i}\right)=g_{\alpha} \frac{\partial K_{i}}{\partial y_{j}}+K_{i} \frac{\partial g_{\alpha}}{\partial y_{j}} .
$$

The first of these is of the same form as above. Hence we can write

$$
\begin{aligned}
H(t- & |y-x|, y, u) \\
= & O\left[g_{\alpha}(t-|y-x|, y, u)\left\{\left|K_{x}(t-|y-x|, y)\right|+D(\varepsilon)(1+t-|x-y|)^{-7 / 4}\right\}\right] \\
& +c(w, \hat{u}) \sum_{i, j} \frac{\partial \hat{u}_{j}}{\partial u_{i}} K_{i}(t-|y-x|, y) \frac{\partial g_{\alpha}}{\partial y_{j}}(t-|y-x|, y, u) .
\end{aligned}
$$

To handle the integral of the last term here, we again replace $\partial / \partial y_{j}$ by appropriate combinations of the operators $T_{j}$ and $S$ beneath (5). Letting $\hat{c}_{i j}(w, \hat{u})=$ $c(w, \hat{u}) \partial \hat{u}_{j} / \partial u_{i}$, we obtain for the integral of the last term in $H$ from (68), the expression

$$
\begin{aligned}
& \int_{|y-x|<t}|x-y|^{-1} \mathscr{X} \sum_{\alpha} e_{\alpha}^{2} m_{\alpha}^{2} \int K_{i} \hat{c}_{i j}\left[\frac{-w_{j}}{1+\hat{u} \cdot w} \nabla_{u} \cdot\left(\frac{e_{\alpha}}{m_{\alpha}} g_{\alpha} K\right)\right. \\
& \left.+\left(\delta_{j l}-\frac{w_{j} \hat{u}_{l}}{1+\hat{u} \cdot w}\right) T_{l} g_{\alpha}\right] d u d y,
\end{aligned}
$$

where $\mathscr{X}=\mathscr{X}(t-|x-y|, y)$, and we employ the summation notation. The first of the terms in (69) is integrated by parts in $u$, resulting in an integral which is estimable as I was (because $\left|\nabla_{u} K\right| \leqq C|B|$ ). The last term in (69) is integrated by parts in $y$. There results a term involving only the data, which is estimated exactly as in Lemma 4, and terms bounded by

$$
\begin{aligned}
& C \int_{|y-x|<t} \mathscr{X}|x-y|^{-1} \int g_{\alpha}(t-|y-x|, y, u)\left|K_{x}(t-|y-x|, y)\right| d u d y \\
& \quad+C \int_{|y-x|<t} \mathscr{X}|y-x|^{-2} \int g_{\alpha}(t-|y-x|, y, u)|K(t-|y-x|, y)| d u d y .
\end{aligned}
$$


Thus by (69), the integral of the last term of $H$ in (68) is dominated for small $\varepsilon$ by

$$
\begin{aligned}
& C D(\varepsilon)(1+k+t+r)^{-3 / 4}(1+k+t-r)^{-7 / 4} \mathscr{X}(t, r) \\
& \quad+C \int_{|y-x|<t} \mathscr{X} \int\left\{|x-y|^{-1} g_{\alpha}\left|K_{x}\right|\right. \\
& \left.\quad+|y-x|^{-2} g_{\alpha}|K|\right\}\left.\right|_{(t-|y-x|, y, u)} d u d y
\end{aligned}
$$

where $D(\varepsilon)=o(1)$ as $\varepsilon \rightarrow 0$.

Now using (70) and (68) in (65), we obtain for small $\varepsilon$

$$
\begin{aligned}
& |\mathrm{II}+\mathrm{III}| \leqq C D(\varepsilon)(1+k+t+r)^{-3 / 4}(1+k+t-r)^{-7 / 4} \mathscr{X}(t, r) \\
& \quad+\sum_{\alpha}\left(C D(\varepsilon) \int_{|y-x|<t} \mathscr{X}|y-x|^{-1}(1+t-|y-x|)^{-7 / 4} \int g_{\alpha}(t-|y-x|, y, u) d u d y\right. \\
& \quad+C \int_{|y-x|<t} \mathscr{X}|y-x|^{-1} \int g_{\alpha}(t-|x-y|, y, u)\left|K_{x}(t-|x-y|, y)\right| d u d y \\
& \left.\quad+C \int \mathscr{X}|y-x|^{-2} \int g_{\alpha}(t-|x-y|, y, u)|K(t-|x-y|, y)| d u d y\right)
\end{aligned}
$$

The second term here is dominated by

$$
C D(\varepsilon) \int_{|y-x|<t} \mathscr{X}|y-x|^{-1}(1+t-|y-x|)^{-19 / 4} d y
$$

where we have used Lemma 3. On the support of $\mathscr{X}$, we have

$$
(1+t-|y-x|)^{-19 / 4} \leqq C(1+k+t-|y-x|+|y|)^{-19 / 4} \text {. }
$$

Hence, using Lemma 17 with $p=19 / 4$ and $q=0$, the second term of (71) is bounded by

$$
\begin{aligned}
& C D(\varepsilon) \int_{|y-x|<t} \mathscr{X}|y-x|^{-1}(1+k+t-|y-x|+|y|)^{-19 / 4} d y \\
& \leqq C D(\varepsilon)(1+k+t+r)^{-1}(1+k+t-r)^{-7 / 4} .
\end{aligned}
$$

Thus the second term of (71) is bounded by the first.

The last term in (71) is, by Lemmas 3 and 12, dominated by

$$
\begin{aligned}
& C \int_{|y-x|<t}|x-y|^{-2} \mathscr{X} D(\varepsilon)(1+k+t-|y-x|+|y|)^{-15 / 4}(1+k+t-|y-x|-|y|)^{-3 / 4} d y \\
& \quad \leqq C \int_{|y-x|<t}|x-y|^{-2} \mathscr{X} D(\varepsilon)(1+k+t-r)^{-3 / 4}(1+k+t-|y-x|+|y|)^{-3} d y \\
& \quad \leqq C D(\varepsilon)(1+k+t+r)^{-1}(1+k+t-r)^{-7 / 4} \mathscr{X}(t, r)
\end{aligned}
$$

by virtue of Lemma 9 .

Therefore the bound (71) simplifies for small $\varepsilon$ to

$$
\begin{aligned}
& |\mathrm{II}+\mathrm{III}| \leqq C D(\varepsilon)(1+t+k+r)^{-3 / 4}(1+k+t-r)^{-7 / 4} \mathscr{X}(t, r) . \\
& \quad+C \int_{|y-x|<t} \mathscr{X}|y-x|^{-1}(1+k+t-|y-x|+|y|)^{-3}\left|K_{x}(t-|x-y|, y)\right| d y,
\end{aligned}
$$

where $D(\varepsilon)=o(1)$ as $\varepsilon \rightarrow 0$ and we have used Lemma 3 again. Our bound for the 
term I above (65) is the same as that displayed in the first term of (74). Summarizing, we have proved

Lemma 18. There exists a function $D_{3}(\varepsilon)$ on $\left(0, \eta_{2}\right)$ into $(0, \infty)$ such that

$$
\begin{gathered}
D_{3}(\varepsilon)=o(1) \quad \text { as } \quad \varepsilon \rightarrow 0 \text {, and } \\
\left|A_{S S}\right| \leqq D_{3}(\varepsilon)(1+k+t+r)^{-3 / 4}(1+k+t-r)^{-7 / 4} \mathscr{X}(t, r) \\
+C \int_{|y-x|<t} \mathscr{X}|y-x|^{-1}(1+k+t-|x-y|+|y|)^{-3}\left|K_{x}(t-|x-y|, y)\right| d y
\end{gathered}
$$

on $0 \leqq t<T_{\varepsilon}$.

Lemma 19. There exists a function $\tilde{D}(\varepsilon)$ on $\left(0, \eta_{2}\right)$ into $(0, \infty)$ such that

$$
\begin{gathered}
\tilde{D}(\varepsilon)=o(1) \quad \text { as } \quad \varepsilon \rightarrow 0, \quad \text { and } \\
\left|\frac{\partial}{\partial x_{k}} E^{i}(t, x)\right| \leqq \tilde{D}(\varepsilon) \mathscr{X}(t, r)(1+k+t+r)^{-3 / 4}(1+k+t-r)^{-7 / 4} \\
+C \int_{|y-x|<t} \mathscr{X}|y-x|^{-1}(1+k+t-|y-x|+|y|)^{-3}\left|K_{x}(t-|x-y|, y)\right| d y
\end{gathered}
$$

on $0 \leqq t<T_{\varepsilon}$.

Proof. By the representation (10) and Lemmas (14)-(18), the result follows immediately.

Now, since the same estimates hold for $\left(\partial / \partial x_{k}\right) B(t, x)$, Lemma 18 yields the inequality

$$
\begin{aligned}
\left|K_{x}(t, x)\right| \leqq & C \tilde{D}(\varepsilon)(1+k+t+r)^{-3 / 4}(1+k+t-r)^{-7 / 4} \mathscr{X}(t, r) \\
& +C \int_{|y-x|<t}|x-y|^{-1}(1+k+t-|y-x|+|y|)^{-3}\left|\left(\mathscr{X} K_{x}\right)(t-|x-y|, y)\right| d y
\end{aligned}
$$

over $0 \leqq t<T_{\varepsilon}$. We can now apply Lemma 11 with the choices $p=3 / 4, q=7 / 4$, $u(t, x)=\left|K_{x}(t, x)\right|$. This gives us the inequality

$$
\left|K_{x}(t, x)\right| \leqq C \tilde{D}(\varepsilon)(1+k+t+r)^{-3 / 4}(1+k+t-r)^{-7 / 4} \mathscr{X}(t, r),
$$

where $0 \leqq t<T_{\varepsilon}, C$ is independent of $t$ and $\tilde{D}(\varepsilon)=o(1)$ as $\varepsilon \rightarrow 0$. Now we observe that from (75) and Lemma 12, the (FSC) holds on $0 \leqq t<T_{\varepsilon}$ whenever $\varepsilon$ is chosen sufficiently small.

Proof of the Main Theorem. From the above, we know that

$$
\left\{\begin{array}{l}
|E|+|B| \leqq \tilde{D}(\varepsilon)(1+k+t+|x|)^{-3 / 4}(1+k+t-|x|)^{-3 / 4} \mathscr{X}(t, x) \\
\left|\nabla_{x} E\right|+\left|\nabla_{x} B\right| \leqq \tilde{D}(\varepsilon)(1+k+t+|x|)^{-3 / 4}(1+k+t-|x|)^{-7 / 4} \mathscr{X}(t, x)
\end{array}\right.
$$

holds for $t \in\left[0, T_{\varepsilon}\right)$, provided $\varepsilon$ is sufficiently small. But recall that $T_{\varepsilon}$ is just the maximal time for which the (FSC) holds with $\eta=\eta_{2}$. Now take $\varepsilon$ sufficiently small that $\widetilde{D}(\varepsilon)<\frac{1}{2} \eta_{2}$. Assume $T_{\varepsilon}$ is finite, then by (76) and continuity we see that the (FSC) actually holds for $t \in\left[0, T_{\varepsilon}+\delta\right.$ ) for some $\delta>0$. This contradicts the maximality of $T_{\varepsilon}$, and hence $T_{\varepsilon}$ must be infinite. Hence the stated decay rates hold for all time and by Lemma 1 the $v$-support of each density $f_{\alpha}(t, x, v)$ is uniformly 
bounded for all $\alpha, x \in \mathbb{R}^{3}, 0 \leqq t<\infty$. This is a sufficient condition for global existence, as we know from [4], and the proof is complete.

\section{References}

1. Bardos, C., Degond, P.: Global existence for the Vlasov-Poisson equation in 3 space variables with small initial data. Internal report no. 101, Centre de Math Appliquees Ecole Polytechnique, Palaiseau (France)

2. Batt, J.: Recent developments in the mathematical investigation of the initial value problem of stellar dynamics and plasma physics. Ann. Nucl. Energy 7, 213-217 (1980)

3. Glassey, R., Schaeffer, J.: On symmetric solutions of the relativistic Vlasov-Poisson system. Commun. Math. Phys. 101, 459-473 (1985)

4. Glassey, R., Strauss, W.: Singularity formation in a collisionless plasma could occur only at high velocities. Arch. Rat. Mech. Anal. 92, 59--90 (1986)

5. Glassey, R., Strauss, W.: Absence of shocks in an initially dilute collisionless plasma. Commun. Math. Phys. 113, 191-208 (1987)

6. Schaeffer, J.: The classical limit of the relativistic Vlasov-Maxwell system. Commun. Math. Phys. 104, 403-421 (1986)

7. Schaeffer, J.: Global existence for the Poisson-Vlasov system with nearly symmetric data. J. Differ. Equations 69, 111-148 (1987)

Communicated by C. H. Taubes

Received September 8, 1987; in revised form April 19, 1988 